\title{
Protective Effects of Notoginsenoside R1 via Regulation of the PI3K- Akt-mTOR/JNK Pathway in Neonatal Cerebral Hypoxic-Ischemic Brain Injury
}

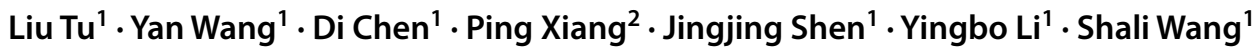

Received: 11 August 2017 / Revised: 22 February 2018 / Accepted: 21 April 2018 / Published online: 25 April 2018

(c) The Author(s) 2018

\begin{abstract}
Notoginsenoside R1 (NGR1) is a predominant phytoestrogen extracted from Panax notoginseng that has recently been reported to play important roles in the treatment of cardiac dysfunction, diabetic kidney disease, and acute liver failure. Studies have suggested that NGR1 may be a viable treatment of hypoxic-ischemic brain damage (HIBD) in neonates by reducing endoplasmic reticulum stress via estrogen receptors (ERs). However, whether NGR1 has other neuroprotective mechanisms or long-term neuroprotective effects is unclear. In this study, oxygen-glucose deprivation/reoxygenation (OGD/R) in primary cortical neurons and unilateral ligation of the common carotid artery (CCL) in 7-day-old postnatal Sprague Dawley (SD) rats followed by exposure to a hypoxic environment were used to mimic an HIBD episode. We assessed the efficacy of NGR1 by measuring neuronal damage with MTT assay and assessed brain injury by TTC staining and brain water content detection 24-48 h after OGD/HIE. Simultaneously, we measured the long-term neurophysiological effects using the beam walking test (5 weeks after HI) and Morris water maze test 5-6 weeks after HI. Expression of PI3K-Akt-mTOR/JNK (24 h after HI or OGD/R) proteins was detected by Western blotting after stimulation with HI, NGR1, LY294002 (PI3K inhibitor), 740Y-P (PI3K agonist), or ICI 182780(estrogen receptors inhibitor). The results indicated that NGR1 exerted neuroprotective effects by inhibiting neuronal apoptosis and promoting cell survival via the PI3K-Akt-mTOR/JNK signaling pathways by targeting ER in neonatal hypoxic-ischemic injury.
\end{abstract}

Keywords Notoginsenoside R1 · Hypoxic-ischemic brain damage · PI3K-Akt-mTOR/JNK signaling pathway · Apoptosis · Estrogen receptors

\section{Introduction}

Hypoxic-ischemic brain damage (HIBD) in neonates is an important risk factor for many severe human neurological dysfunctions, such as motor and learning disabilities, cerebral palsy, epilepsy, and even death [1-3]. In spite of the major advances in modern medical technology and the increased understanding of fetal and neonatal pathologies, neonatal hypoxic-ischemic encephalopathy (HIE) is still

Shali Wang

shaliwang2017@163.com

1 Cerebrovascular Diseases Laboratory, Institute of Neuroscience, Chongqing Medical University, No. 1, Yixueyuan Road, Yuzhong District, Chongqing 400016, China

2 Department of Cardiology, Children's Hospital of Chongqing Medical University, Chongqing, China an unresolved serious condition that leads to significant mortality and long-term morbidity [4-7]. Presently, there are no well-established effective therapies for neonatal HIE [8]. Hypoxic-ischemic brain injury directly results in a large amount of neuronal death. Research suggested that an important way causing neuronal loss was apoptosis, especially in the penumbra area [9]. Malagelada et al. [10] found that there were at least $50 \%$ of dying cells which performed morphological characteristics of apoptosis in OGD-treated cortical neuron cultures. Therefore, enhancing neuronal survival, reducing apoptosis have become the most important strategies for solving neurological diseases [11].

Notoginsenoside R1 (NGR1) is a predominant phytoestrogen extracted from $P$. notoginseng. NGR 1 was recently reported to possess anti-inflammatory, antioxidant, and antiapoptotic properties, and may play important roles in the treatment of cardiac dysfunction [12-15], acute liver failure [16], and diabetic kidney disease [17]. Meng et al. [18] found 
that 3-day pretreatment with NGR1 significantly reduced cerebral infarct volume in an adult rat model, while pretreatment with NGR1 for $24 \mathrm{~h}$ prevented apoptosis induced by oxygen glucose deprivation/reoxygenation (OGD/R) in primary cortical neurons. Our past study [19] indicated that NGR 1 treatment exerted neuroprotective effects in the acute phase of a neonatal HIBD model. It is worth noting that neonatal HIBD often leads to long-lasting neurological deficits such as mental deficiency, cerebral palsy, and learning disabilities, which develop in the immature brain. These consequences have seriously affected the quality of life of children with HIE. Whether NGR1 treatment can promote the long-term recovery of neurological function after HIBD has not yet been reported and is worth exploring.

Research $[13,15,18,19]$ has indicated that NGR1 may perform its functions through estrogen receptors (ERs). The classic ERs have two major subunits, estrogen receptor $\alpha(\mathrm{ER} \alpha)$ and estrogen receptor $\beta(\mathrm{ER} \beta)$. Within the brain, $E R \alpha / \beta$ are found in cognitive brain regions associated with learning and memory, such as the cerebral cortex, hippocampus, and basal forebrain [20,21]. A number of studies have shown that ERs play an important role in organ ischemic injury. Liu et al. [22] found that calycosin exhibited an anti-apoptotic effect via $E R \alpha / \beta$ and improved Akt phosphorylation in cardiomyocytes. Hsu et al. [23] suggested that $17 \beta$-estradiol (E2) treatment reversed hepatic injury following hemorrhagic shock and resuscitation through ERsrelated p38 MAPK-dependent HO-1 upregulation. Wang et al. [24] reported that $\mathrm{E} 2$ offered protection against retinal ischemic injury by inducing upregulation of SDF-1 expression through activation of ERs. Activating ERs were found to provide protection for CA1 neurons in ischemic injury, while ICI 182780 (the broad-spectrum ERs antagonist) abolished the protection [25].

As an important signal transduction pathway, PI3KAkt-mTOR/JNK is involved in many cellular processes, including cell apoptosis, survival and proliferation [26, 27]. Phosphatidylinositol 3 kinase (PI3K) is an intracellular phosphatidylinositol kinase which consists of a catalytic subunit (p110) and a regulatory subunit (p85) [28, 29]. Protein kinase B (Akt), a serine/threonine kinase, is a primary downstream target in the transduction pathway of PI3K signaling. Akt is a key information molecule that promotes cell survival, inhibits apoptosis [30] and maintains normal functions [31]. Activated Akt can transmit signals to a variety of downstream substrates. The common downstream proteins include TSC1/2-Rheb-mTOR [32], pro-apoptotic factor JNK, NFkb, and frontal transcription factor FKHR [33]. Mammalian target of rapamycin (mTOR) is a serine/ threonine kinase that can benefit cell growth, survival, and metabolism [32]. The main targets of activated mTOR are ribosomal protein S6 kinase (p70S6K) and eukaryotic initiation factor $4 \mathrm{E}$ binding protein 1 (4E-BP1). Among them,
p70S6K is mainly involved in cell-cycle regulation and contributes critically to cell survival. Activated p70S6K promotes the synthesis of ribosome translation regulator protein, resulting in the positive regulation of protein synthesis. Through the phosphorylation of 4E-BP1, mTOR regulates cap-dependent protein translation and promotes the proliferation of neurons. JNK which also can be regulated by Akt directly or indirectly controls a number of transcriptional and non-transcriptional processes, including inflammation and cell death or survival [26, 34-39].

Many studies have shown that PI3K-Akt-mTOR/JNK signaling plays a major role in cerebral hypoxic-ischemic injury [26, 32, 40, 41]. Some researchers [42-44] have found that Akt signaling, which is activated after transient cerebral ischemia, inhibits delayed neuronal apoptosis and promotes cell survival. Activation of the mTOR pathway is sufficient for promoting both neuron survival and axon regeneration [45, 46]. Research [26, 47, 48] indicates that the JNK pathway is also involved in ischemia-induced neuronal apoptosis. Hence, a number of researchers have proposed that JNK may be a target for the treatment of neuronal necrosis and that the inhibition of the JNK signaling pathway may reduce the apoptosis caused by ischemic brain damage [49-51].

Some studies have reported that NGR1 could protect the heart from septic shock via the activation of ER $\alpha$ and PI3K/ Akt signaling [13]. NGR1 activated Nrf2/ARE signaling and upregulated phase II antioxidant enzymes in PC12 cells via ERs [52]. Our previous findings suggested that NGR1 could inhibit endoplasmic reticulum stress-induced neuronal apoptosis and brain damage via ERs [19]. However, it remained unclear whether NGR1 could exert neuroprotective effects and reduce neuron apoptosis via ERs by acting on the PI3K-Akt-mTOR/JNK signal pathway in a neonatal hypoxic-ischemic brain damage (HIBD) model.

In this study, we investigated the neuroprotective effects of NGR1 in a neonatal HIBD model, especially concerned whether NGR1 had a contribution to the long-term recovery of neurological function in the HIE. Furthermore, we explored the neuroprotective mechanisms of NGR1 by inhibiting neuronal apoptosis and promoting cell survival via the ERs and PI3K-Akt-mTOR/JNK signaling pathway.

\section{Materials and Methods}

\section{Drug Preparation}

NGR1 (chemical structure $\mathrm{C}_{47} \mathrm{H}_{80} \mathrm{O}_{18}$, molecular weight $=933.13$, purity $>98 \%$ ) was from Sigma-Aldrich (Sigma-Aldrich, St. Louis, MO). ICI-182780 (an estrogen receptor inhibitor), LY294002 (an inhibitor of PI3K) and 740Y-P (an agonist of PI3K) were from Tocris (London, 
UK), Selleck Chemicals (Houston, Texas, USA), and Selleck Chemicals (Houston, Texas, USA), respectively.

\section{Animals}

Seven-day-old Sprague-Dawley (SD) male rats and rat fetuses (18 days) were provided by the Animal Department of Chongqing Medical University (Chongqing, China). All experiments were put into practice in accordance with the National Institutes of Health Guide for the Care and Use of Laboratory Animals. All protocols were ratified by the Animal Ethics Committee of Chongqing Medical University and efforts were made to reduce animal suffering.

\section{Cell Culture and Drug Treatment}

The experiment was conducted according to previously described methods $[19,53]$. Dissociated cultures of cortical neurons were harvested from time-mated embryonic day 18 (E18) rat brains using established protocols. Cerebral cortices were excised and hatched in $\mathrm{Ca}^{2+}$ - and $\mathrm{Mg}^{2+}$-free HBSS solution. The tissues were mechanically separated and then digested in $0.25 \%$ trypsin (with $0.02 \%$ EDTA) for $7 \mathrm{~min}$ at $37{ }^{\circ} \mathrm{C}$. After trypsinization was terminated, the digests were centrifuged for $5 \mathrm{~min}$ at $1000 \mathrm{rpm}$. The centrifuged cells were resuspended in Neurobasal medium (Gibco, Gaithersburg, MD) with 2\% B-27 supplement (Gibco) and $2 \mathrm{mmol} / \mathrm{l} \mathrm{L}$-glutamine (Invitrogen, Gaithersburg, MD). Cells were subcultured in 96-well plates $\left(5 \times 10^{4}\right.$ cells/well) for 3-(4,5-dimethyl-2-thiazolyl)-2,5-diphenyl-2H-tetrazolium bromide (MTT) assays, in 24-well plates $\left(1 \times 10^{5}\right.$ cells/ well) for lactate dehydrogenase (LDH) determination and in 6-well plates $\left(1 \times 10^{6}\right.$ cells/well $)$ for other experiments. Plates were precoated with polyethylenimine $(0.05 \mathrm{mg} / \mathrm{ml}$, Sigma-Aldrich) overnight at $37{ }^{\circ} \mathrm{C}$. Cultures were maintained in a Heraeus $\mathrm{CO}_{2}$ incubator (Thermo Fisher Scientific, Rockford, IL) containing $5 \% \mathrm{CO}_{2}$ and $95 \%$ air at $37^{\circ} \mathrm{C}$. Cultures were used for experiments on the fifth day in vitro. Cells were treated with NGR1 $(10 \mu \mathrm{mol} / \mathrm{l})$ [19] when subjected to oxygen glucose deprivation and reoxygenated. ICI $182780(0.1 \mu \mathrm{mol} / \mathrm{l})$ [19] was used to preprocess cells $2 \mathrm{~h}$ before OGD. LY294002 $(20 \mu \mathrm{mol} / \mathrm{l})$ and 740Y-P $(20 \mu \mathrm{mol} / \mathrm{l})$ were applied to cells $1 \mathrm{~h}$ before OGD. ICI 182780, LY294002, and 740Y-P were dissolved in dimethyl sulfoxide (DMSO). DMSO acted as a vehicle with a concentration of $1 \%$.

\section{Oxygen Glucose Deprivation/Reoxygenation}

OGD/R was accomplished using day-5 cultured primary cortical neurons to imitate cerebral ischemic/reperfusion injury. OGD/R was achieved using a modification of a previously described procedure [19]. After the cells were washed once with phosphate-buffered saline (PBS), culture plates were replenished with glucose-free Dulbecco's Modified Eagle's Medium. Cultures were placed in an anaerobic chamber (Thermo Fisher Scientific) and incubated in an anaerobic gas mixture $\left(1 \% \mathrm{O}_{2}, 5 \% \mathrm{CO}_{2}\right.$, and $\left.94 \% \mathrm{~N}_{2}\right)$ at $37{ }^{\circ} \mathrm{C}$. After $1.5 \mathrm{~h}$, cultures were returned to a normoxic environment from the anaerobic chamber. Simultaneously, the culture plates were refilled with Neurobasal medium, and cultures were allowed to reoxygenate for $4-24 \mathrm{~h}$.

\section{Hypoxic-Ischemic Brain Damage Model}

HI was imitated by unilateral ligation of the common carotid artery (CCL) followed by $2.5 \mathrm{~h}$ of hypoxia in 7-day-old SD rats. Rat pups were anesthetized with isoflurane $(2.5 \%)$ and supine fixed in the thermostat console. A longitudinal midline incision disinfected by iodophor disinfectant was made in the anterior neck. After the right common carotid artery was identified and freed from the surrounding tissues, without any damage to the right vagus nerve, it was double ligated and transected between the ligatures. The pups were then returned to a heating pad for $1 \mathrm{~h}$ for recovery. Simultaneously, an airtight chamber containing $7 \%$ humidified oxygen and $93 \% \mathrm{~N}_{2}$ was prepared using a heating pad to maintain the temperature at $35-39{ }^{\circ} \mathrm{C}$. Then the $\mathrm{HI}$ animals were placed in the chamber for $2.5 \mathrm{~h}$. Sham animals received an incision but did not undergo CCL treatment, and the pups were placed in a similar container but not exposed to a hypoxic environment. After modeling, all pups were returned to their dams. NGR1 ( $15 \mathrm{mg} / \mathrm{kg} \mathrm{q} 12 \mathrm{~h}$, for 2 days) [19] was administered to the pups by intraperitoneal injection after CCL immediately, before exposure to the hypoxic environment. ICI-182780 (2 mg/ $\mathrm{kg})$ was administered to pups $2 \mathrm{~h}$ before CCL treatment by intraperitoneal injection [19].

\section{Cell Viability Assessment}

An MTT assay was used to test cell viability. Four or $24 \mathrm{~h}$ after the OGD/R injury, cells were incubated with MTT $(0.05 \mathrm{mg} / \mathrm{l})$ for $4 \mathrm{~h}$ at $37^{\circ} \mathrm{C}$. The culture medium was then completely removed, and all wells were filled with $100 \mu \mathrm{l}$ DMSO to dissolve the formazan crystals. Absorbance was surveyed at $570 \mathrm{~nm}$ using a microplate reader (Bio-Rad Model 680, Bio-Rad, Hercules, CA). Cell viability was calculated using the formula (mean experimental absorbance/ mean control absorbance) $\times 100 \%$.

\section{Measurement of Cell Membrane Integrity}

The rate of LDH release was used to estimate the membrane integrity of cells. The supernatant of each well was collected, and the LDH content was determined using an 
LDH assay kit according to the manufacturer's instructions (Nanjing Institute of Jiancheng Biologic Engineering, Nanjing, China). For the positive control, the supernatant of the cells was collected after cells were lysed using $0.25 \%$ Triton $\mathrm{X}-100$. The level of LDH release was calculated using the formula (experimental LDH activity/positive control LDH activity) $\times 100 \%$.

\section{Morris Water Maze}

Neurocognitive outcomes were measured by using the Morris water maze (WM) test with a computerized video tracking system (BW-mwm101, Shanghai BioWill Co., Ltd., China) 5-6 weeks after modeling. The WM consisted of a circular pool $120 \mathrm{~cm}$ in diameter and $47 \mathrm{~cm}$ in height, containing water $30 \mathrm{~cm}$ deep. A hidden submerged platform ( $9 \mathrm{~cm}$ diameter) was placed in the second quadrant $2.5 \mathrm{~cm}$ below the water surface for rats to step on and escape from the water. Rats could identify the position of the platform using visual clues placed on the walls. The time to locate the submerged platform (defined as the latency, with cutoff time $60 \mathrm{~s}$ ) was measured. Every day, each rat performed four trials starting from different quadrants. The test lasted for 5 days. On testing day 6 , each rat performed a probe trial ( $60 \mathrm{~s}$ cutoff) without a platform. All of the activities were video recorded, and the animals' swimming paths were measured for quantification of time, frequency, and latency $[54,55]$ using the ANY-maze Animal Behavioral Video Analysis System (Shanghai Bio-will Co., Ltd, China).

\section{Beam Walking Test}

Coordination and integration of motor movement was assessed with a beam $(80 \mathrm{~cm} \times 2.0 \mathrm{~cm} \times 2.5 \mathrm{~cm} ; 60 \mathrm{~cm}$ above floor) walking test 5 weeks after modeling. Each rat was tested 3 times, for 2 min each time. The ratio scale was modified from Ohlsson [56] and Feeney [57]. Balance performance on the beam was graded as follows: 0 , the rat falls down and cannot walk on the beam; 1 , the rat is unable to walk on the beam but can sit on the beam; 2 , the rat falls down while walking; 3 , the rat can traverse the beam, but the affected hind limb does not aid in forward locomotion; 4 , the rat crosses the beam with more than $50 \%$ foot slips; 5 , the rat traverses the beam with fewer than $50 \%$ foot slips; 6 , the rat successfully crosses the beam with no foot slips.

\section{Evaluation of Brain Damage 6 Weeks After Modeling}

Hemispheric weight loss has been used as an important variable for assessing brain atrophy in neonatal HI model [58]. After Morris water maze test, the brains were extracted and the hemispheres were cut along the center line and weighed on a high-precision balance. The brain weight ratio (\%) was calculated using the formula (weight of ipsilateral hemisphere/weight of contralateral hemisphere $) \times 100 \%$.

\section{Evaluation of Infarction Volume}

2,3,4-tiphenyl tetrazolium chloride (TTC) (Sigma-Aldrich, $\mathrm{MO}$ ) staining is a reliable way to evaluate infarction volume. Using this method, the brain sections were prepared as follows: First, the brains were removed and frozen at $-20{ }^{\circ} \mathrm{C}$ for $10 \mathrm{~min}$. Next, consecutive $2 \mathrm{~mm}$ coronal sections were obtained by slicing the brains with Brain Matrix (ASI Instruments, Warren, MI). The subsequent incubation of the sections was performed in a dark environment with 25 -min immersion in $2 \%$ TTC solution at $37^{\circ} \mathrm{C}$. Finally, the sections were immersed in a $4 \%$ formaldehyde solution. TTC stained normal areas of brain deep red but did not stain infarcted tissue. Infarction volumes were measured and analyzed with ImageJ software (NIH Image, Version 1.61, Bethesda, MD, USA) as described previously [19].

\section{Brain Water Content Detection}

Rats were sacrificed $24 \mathrm{~h}$ after HI for brain water content measurement. The wet weight of the brain sample was measured immediately after harvest. The brain was then placed in an oven at $105^{\circ} \mathrm{C}$ for $24 \mathrm{~h}$ and weighed again to determine the dry weight [59]. Brain water content (\%) was calculated using the formula[(wet weight - dry weight)/wet weight $] \times 100 \%$.

\section{TUNEL Staining}

Coronal brain slices were stained with neuron-specific nuclear protein (NeuN) and terminal deoxynucleotidyl transferase-mediated nick-end labeling (TUNEL) to measure apoptotic neurons $24 \mathrm{~h}$ after HI. After dewaxing by xylene, sections were subjected to gradient hydration. The slices were incubated with anti-NeuN (1:50, Abcam) and Alexa Fluor 555-labeled goat anti-mouse IgG (1:100, Beyotime Institute of Biotechnology). Afterward, samples were added to the TUNEL reaction mixture (Thermo Fisher Scientific) for an incubation time of $60 \mathrm{~min}$ at $37{ }^{\circ} \mathrm{C}$ in a humidified atmosphere in the dark. Then, DAPI was used to incubate the samples for $2 \mathrm{~min}$. Apoptotic cells were photographed under a microscope (Olympus) with an excitation wavelength of 450-500 $\mathrm{nm}$ (green) and a detection wavelength of 515-565 nm (red). Three coronal brain sections were selected from each brain (six animals in each group), and the numbers of positive cells (neurons) in the ipsilateral cerebral cortex was counted for each section at high magnification in five visual fields. The proportion of TUNEL-positive cell nuclei was determined by dividing the number of TUNELpositive nuclei by the number of total nuclei. 


\section{Western Blots}

Protein expression was evaluated through Western blot analysis. Cells or brain tissues (Respectively taking the contralateral hemisphere and ipsilateral hemisphere) were homogenized by lysis buffer (Beyotime Institute of Biotechnology). The insoluble material was removed by centrifugation at $12,500 \mathrm{rpm}$ for $15 \mathrm{~min}$ at $4{ }^{\circ} \mathrm{C}$. The supernatants of the lysate were collected to measure the protein concentration with a BCA Protein Assay Kit (Thermo Fisher Scientific). Protein samples were denatured for $5 \mathrm{~min}$ at $100{ }^{\circ} \mathrm{C}$ after being mixed with sodium SDS gel-loading buffer. Then, samples were separated by SDS-polyacrylamide gel electrophoresis and transferred to a polyvinylidene membrane (the specific conditions of electrophoresis and transfer varied according to the molecular weight of the target protein). Membranes were blocked for $2 \mathrm{~h}$ in 5\% nonfat dry milk in Tween/Tris-buffered saline (TTBS) at room temperature. The membranes were then incubated with the primary antibody. After incubation overnight at $4{ }^{\circ} \mathrm{C}$, the membranes were washed with Tris-buffered saline and incubated with a secondary antibody for about $2 \mathrm{~h}$ at room temperature. Bands were scanned and densitometrically analyzed by automated ImageJ software (NIHImage, Version 1.61).

\section{Statistical Analysis}

All data are expressed as mean \pm SEM statistical analyses were carried out by SPSS version 17.0 (SPSS, Chicago, IL). One-way analysis of variance was used to evaluate the significance of differences among experimental groups. A $p$ value of 0.05 was regarded as the level of statistical significance.

\section{Results}

\section{NGR1 Attenuated OGD/R-Induced Cortical Neuron Damage Mediated by Estrogen Receptors}

As the main component of the phytoestrogen from $P$. notoginseng, NGR1 protected the cortical neurons from injury induced by OGD/R, but this effect could be blocked by ERs blocker ICI 182780. Neuronal damage was measured by MTT assay and LDH leakage performed at 4 or $24 \mathrm{~h}$ after OGD/R (Fig. 1). The results showed that NGR1 (10 $\mu \mathrm{mol} / \mathrm{l})$ significantly improved neuronal cell viability $(83.17 \pm 13.68$ vs. $65.71 \pm 13.60 \%, p<0.05$, at $4 \mathrm{~h}$ after $\mathrm{OGD} / \mathrm{R}$; $86.01 \pm 9.17$ vs. $62.85 \pm 18.31 \%, p<0.05$, at $24 \mathrm{~h}$ after $\mathrm{OGD} / \mathrm{R})$ and reduced the $\mathrm{LDH}$ leakage rate $(19.23 \pm 3.24$ vs. $26.92 \pm 5.86 \%, p<0.05$, at $4 \mathrm{~h}$ after OGD/R; $28.31 \pm 8.34$ vs. $39.75 \pm 10.20 \%, p<0.05$, at $24 \mathrm{~h}$ after OGD/R) in the cortical neuron OGD/R model compared with the OGD/R group.
However, ICI 182780 could suppress these neuroprotective effects of NGR1. In the OGD/R + NGR $1+$ ICI 182780 group, the cell viability was significantly reduced $(67.19 \pm 14.28$ vs. $83.17 \pm 13.68 \%, p<0.05$, at $4 \mathrm{~h}$ after $\mathrm{OGD} / \mathrm{R} ; 65.81 \pm 17.36$ vs. $86.01 \pm 9.17 \%, p<0.05$, at $24 \mathrm{~h}$ after OGD/R), and the LDH leakage rate was significantly increased $(25.18 \pm 4.76$ vs. $19.23 \pm 3.24 \%, p<0.05$, at $4 \mathrm{~h}$ after OGD/R; $39.36 \pm 8.02$ vs. $28.31 \pm 8.34 \%, p<0.05$, at $24 \mathrm{~h}$ after OGD/R) compared with the OGD/R + NGR1 group. There was no significant difference in cell viability or LDH leakage rate between the DMSO vehicle group and the OGD/R group.

\section{NGR1 Attenuated HI-Induced Brain Injury in Newborn Rats Mediated by Estrogen Receptors}

Brain edema was detected at $24 \mathrm{~h}$ after HI (Fig. 2a), as indicated by increased brain water content. Compared with the sham group $(85.46 \pm 2.43 \%)$, the ipsilateral hemisphere water content was significantly increased in the HI group $(93.36 \pm 3.41 \%, p<0.001$ vs. the sham group). The ipsilateral hemisphere water content was significantly reduced by treatment with NGR1 $(90.12 \pm 2.78 \%, p<0.05$ vs. the HI group), but this effect could be reversed by ICI 182780 $(93.09 \pm 2.63 \%, p<0.05$ vs. the HI + NGR1 group).

Infarct volume was used to evaluate brain damage at $48 \mathrm{~h}$ after HI injury. As shown in Fig. 2b, c, HI caused an increased magnitude of infarction in the right hemisphere $(34.49 \pm 9.49 \%)$, and the infarct volume was significantly reduced in the HI + NGR 1 group $(22.49 \pm 11.63 \%, p<0.01$ vs. the HI group). The result supported the neuroprotective effect of NGR1. Quantitative comparisons of the infarct volumes of the $\mathrm{HI}+\mathrm{NGR} 1$ group and the $\mathrm{HI}+\mathrm{NGR} 1+\mathrm{ICI}$ 182780 group showed that the degree of infarction was intensified in the latter $(31.74 \pm 8.90 \%, p<0.05$ vs. the $\mathrm{HI}+\mathrm{NGR} 1$ group).

The cortical neuronal apoptosis was observed at $24 \mathrm{~h}$ after HI injury. Few TUNEL-positive cortical neurons were found in the sham group, while in the HI group, neuronal apoptosis was $37.35 \pm 10.16 \%$. In comparison, neuronal apoptosis was $21.10 \pm 11.00 \%$ in the HI + NGR1 group ( $p<0.01$ vs. the HI group), however the neuroprotective effect of NGR1 could be reversed by ICI $182780(33.48 \pm 9.53 \%, p<0.05$ vs. the $\mathrm{HI}+\mathrm{NGR} 1$ group) (Fig. 2d, e).

In order to observe the long-term effect of NGR1 on HIBD, the hemisphere weight was estimated at 6 weeks after surgery [38]. The HI injury caused severely brain atrophy, marked by a decrease in the right-to-left hemispheric weight ratio in $\mathrm{HI}$ group $(0.35 \pm 0.20, p<0.001$ vs. the sham group), but the brain atrophy was significantly improved in the HI + NGR 1 group $(0.64 \pm 0.18, p<0.01$ vs. the HI group) (Fig. 2f). Blockage of ERs reversed the neuroprotective effect $(0.48 \pm 0.19, p<0.05$ vs. the HI $+\mathrm{NGR} 1$ group $)$. 

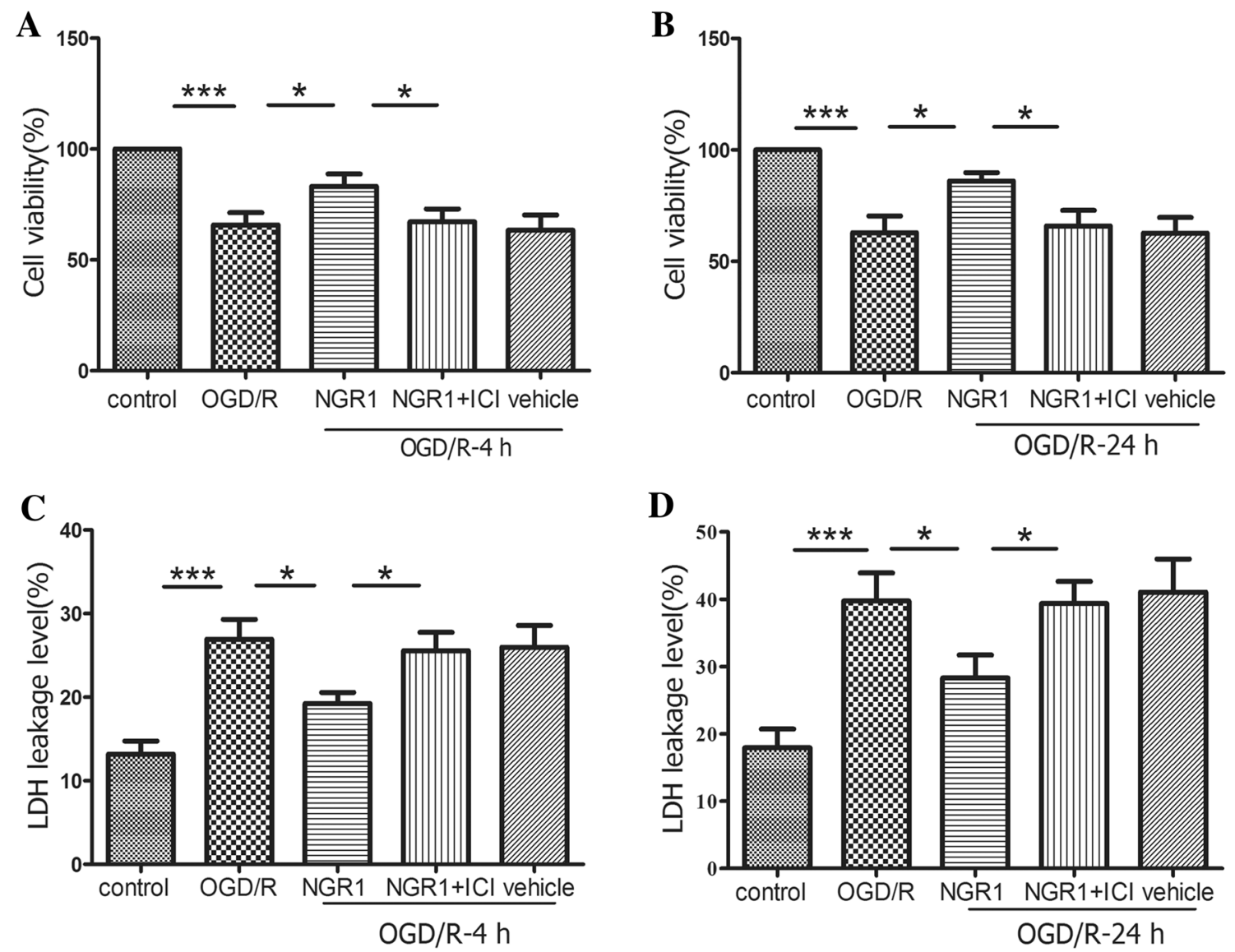

Fig. 1 The effects of NGR1 treatment on neuron injury after OGD/R via estrogen receptors. a and b At 4 and $24 \mathrm{~h}$ after OGD/R, NGR1 increased cell viability compared with the OGD/R group, ICI 182780 pretreatment could abolish this effects. The OGD/R + NGR $1+$ ICI 182780 group had lower cell viability compared with the

\section{NGR1 improved neurobehavioral function Mediated by Estrogen Receptors}

Balance performance was severely impaired in the HI group at 5 weeks after $\mathrm{HI}$ insult (Fig. 3a). In contrast, rats treated with NGR1 showed significantly improved balance performance compared with the HI group $(3.44 \pm 1.01$ vs. $2.33 \pm 1.12, p<0.05)$. However, the protective effect of NGR1 was blocked by ICI 182780 . The result showed significantly reduced scores in the HI + NGR1 + ICI 182780 group $(2.56 \pm 1.13, p<0.05$ vs. the HI + NGR 1 group $)$.

NGR1 could improve spatial learning and memory function recovery, as indicated by the Morris water maze test which was detected 5-6 weeks after neonatal HI injury. The rats' escape latency reflected their spatial learning and memory impairments. The results (Fig. 3b-f) showed that the latencies of the sham group were significantly shortened after 2 days of training, which indicated that the sham group rats had intact learning and memory

OGD/R + NGR 1 group. $\mathbf{c}$ and d At 4 and $24 \mathrm{~h}$ after OGD/R, NGR1 treatment reduced LDH release in neurons and ICI 182780 reversed this effects. Data are expressed as the mean \pm SEM for $\mathrm{n}=6$. $* p<0.05 ; * * p<0.01 ; * * * p<0.001$

capacities. At the end of the fifth day of training, almost all rats could aim to move in the direction of the platform. After the platform was removed, some sham group rats went directly to the location of the platform and wandered nearby, which suggested that the rats had remembered the location of the platform. However, the HI group rats mostly swam in the pool without showing obvious signs of proximity to the platform. The latencies of the HI group in each of the four quadrants were $50.11 \pm 15.19$, $40.23 \pm 15.53,38.43 \pm 13.32,39.89 \pm 15.46 \mathrm{~s}$, respectively. They were higher than those of the sham group $(13.21 \pm 7.70,4.98 \pm 4.20,5.12 \pm 3.46$, and $5.01 \pm 4.88 \mathrm{~s}$, respectively; $p<0.05$ vs. the HI group). Moreover, in the sham group, the percentage (Fig. $3 \mathrm{~g}$ ) of time spent in the target quadrant $(55.02 \pm 12.90$ vs. $24.78 \pm 11.13 \%$, $p<0.001)$ and the frequency $(4.56 \pm 1.32$ vs. $0.75 \pm 0.77$, $p<0.001$ ) of crossing the target platform (where the platform was previously located) were significantly higher than in the HI group (Fig. 3h). These results indicated 
A

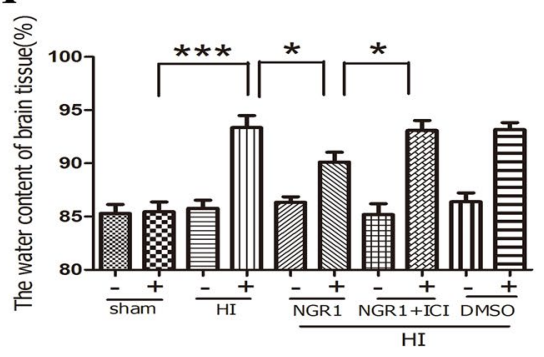

C

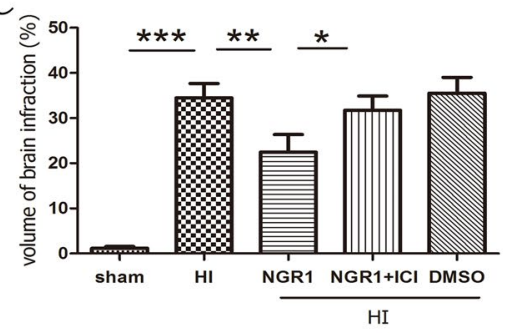

$\mathbf{E}$
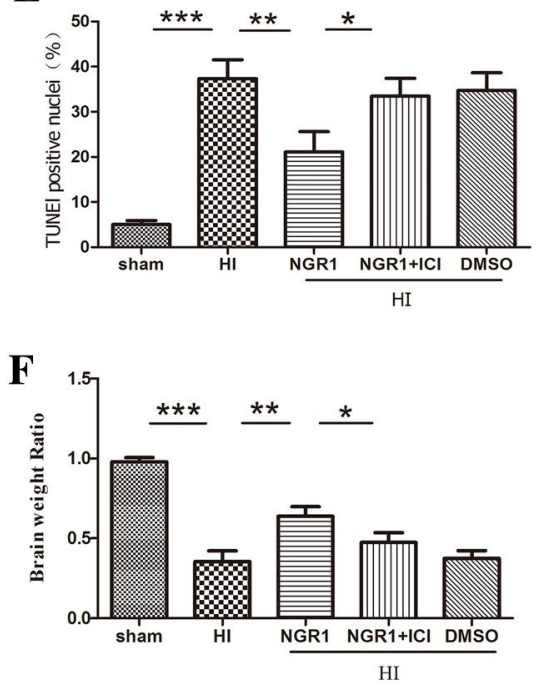

B

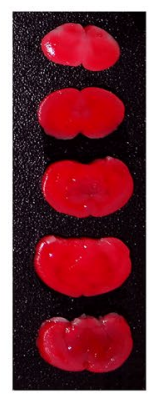

sham

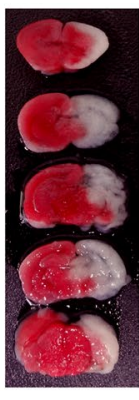

$\mathrm{HI}$

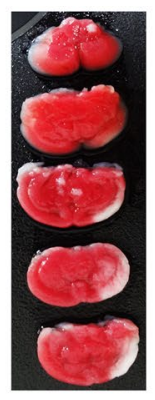

HI+NGR1

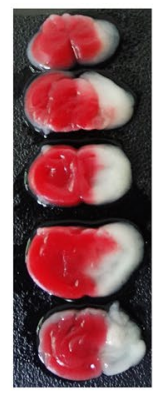

HI+NGR1 $+\mid$ C1182780

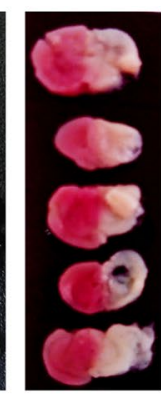

HI+DMSO
D
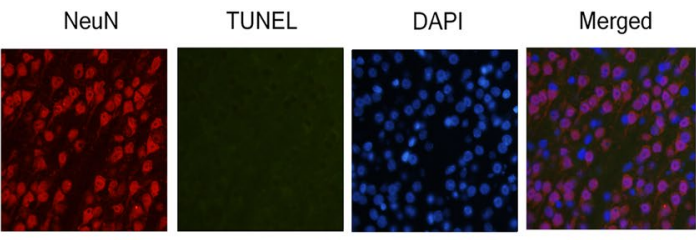

$\mathrm{HI}$
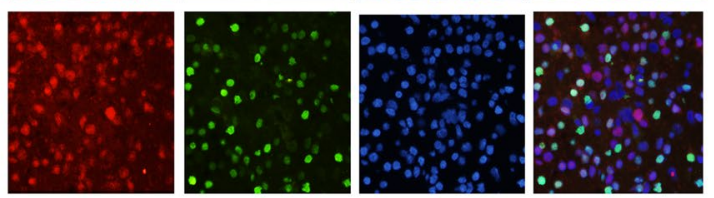

HI+NGR1
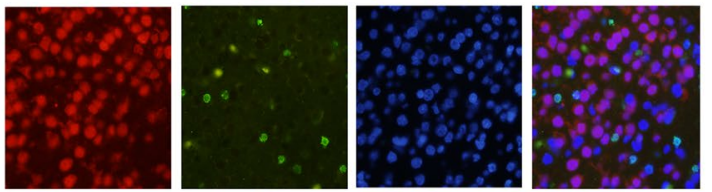

HI+NGR1

$+\mid \mathrm{Cl} 182780$
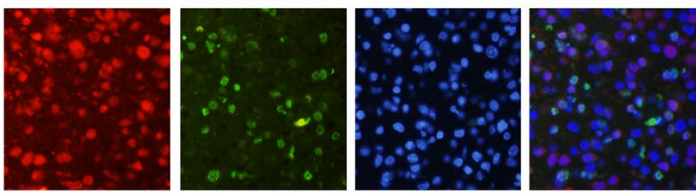

HI+DMSO
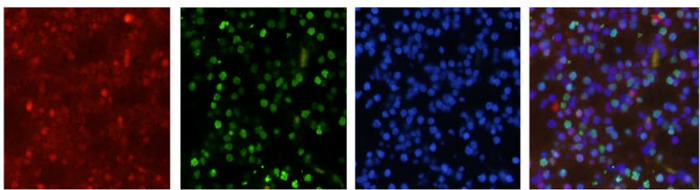

Fig. 2 The effects of NGR1 on brain injury after HI via estrogen receptors. a The water content in the ipsilateral hemisphere was significantly decreased in the NGR1 treatment group compared with the HI group. There was also a significant increase in water content in the HI+NGR1 + ICI 182780 group compared with the HI + NGR1 group. (sham $\mathrm{n}=7, \mathrm{HI} \mathrm{n}=9$, NGR1 $\mathrm{n}=9$, HI+NGR1+ICI 182780 $\mathrm{n}=8, \mathrm{HI}+\mathrm{DMSO} \mathrm{n}=7$; + means ipsilateral, - means contralateral). b and $\mathbf{c}$ NGR 1 could reduce the infarction area, but the neuroprotective effect was blocked by ICI 182780. The HI + NGR1 + ICI 182780 group showed a larger infarction area than the NGR1 treatment group (sham $\mathrm{n}=6, \mathrm{HI} \mathrm{n}=9$, NGR1 $\mathrm{n}=9$, HI+NGR1+ICI $182780 \mathrm{n}=8$, $\mathrm{HI}+\mathrm{DMSO} \mathrm{n}=7$ ). $\mathbf{d}$ and e The number of TUNEL-positive corti- cal neurons were greater in the HI group than in the HI+NGR1 group, but the administration of ICI 182780 could inhibit the protective effect of NGR1. A large number of TUNEL-positive cortical neurons were also found in the HI+NGR1+ICI 182780 group $(n=6)$. Data are expressed as mean \pm SEM. $f$ The ipsilateral hemisphere weight was significantly decreased in the HI group compared with the NGR1 treatment group 6 weeks after HI. ICI 182780 could block this effect. There was also a significant reduction of ipsilateral hemisphere weight in the HI+NGR1+ICI 182780 group compared with the HI+NGR1 group (sham $n=8, H I n=9, H I+N G R 1$ $\mathrm{n}=9, \mathrm{HI}+\mathrm{NGR} 1+\mathrm{ICI} 182780 \mathrm{n}=9, \mathrm{HI}+\mathrm{DMSO} \mathrm{n}=9) . * p<0.05$; $* * p<0.01 ; * * * p<0.001$ that the spatial learning and memory function of HI group rats had been severely weakened as a result of the injury. NGR1 showed neuroprotective effects by significantly decreasing the rats' latencies $(33.43 \pm 13.23,20.57 \pm 9.90$, $20.78 \pm 8.78$, and $27.44 \pm 11.43 \mathrm{~s}$, respectively; $p<0.05$ vs. the HI group) and increasing the percentage of time spent in the target quadrant $(36.51 \pm 13.49 \%, p<0.01$ vs. the HI group) and the frequency of crossing the target platform $(1.72 \pm 1.09, p<0.01$ vs. the HI group). However, the protective effects could be reversed by ICI 

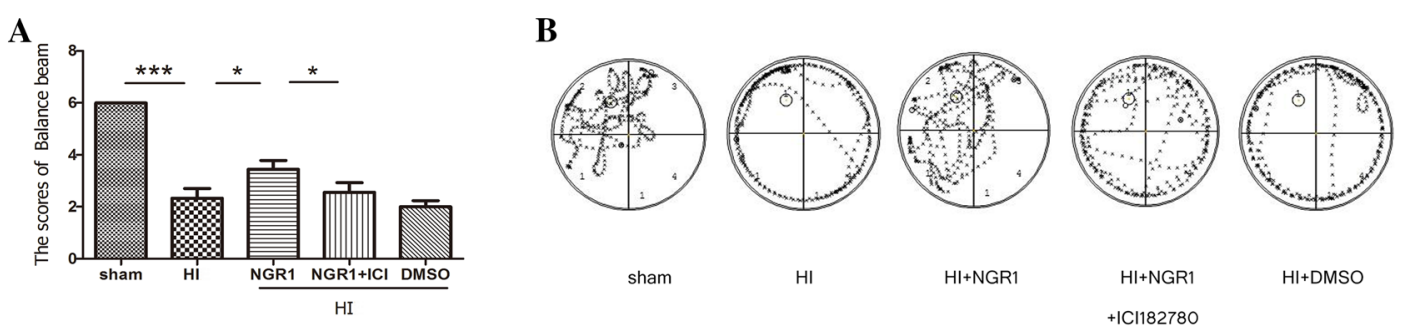
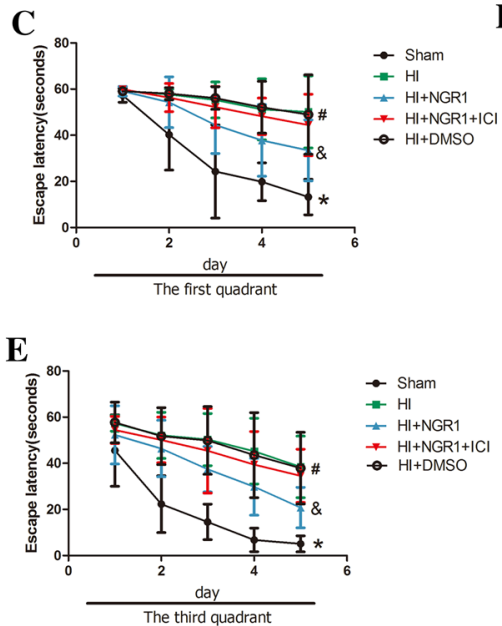

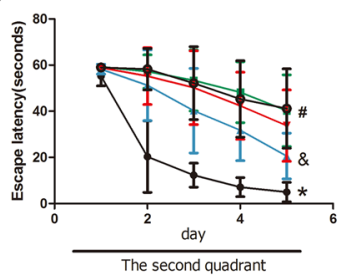

F

B

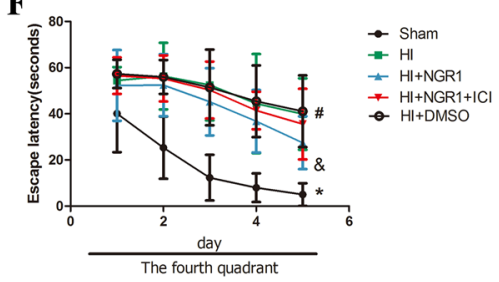

G

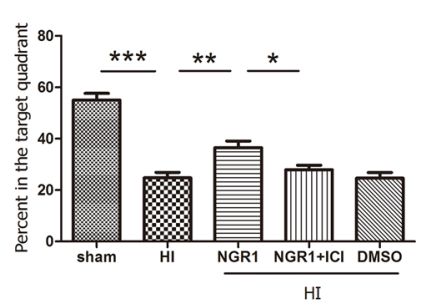

H

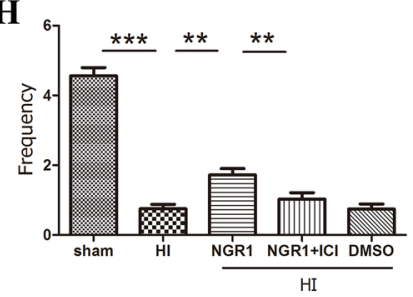

Fig. 3 Neurobehavioral effects of NGR1 5-6 weeks after HI via estrogen receptors. a Balance performance was severely impaired in the HI group at 5 weeks after HI, but NGR1 treatment significantly improved balance performance. The protective effect of NGR1 was blocked by ICI 182780 . b-h The Morris water maze test was performed 5-6 weeks after HI. The results showed that the latencies of the HI group were significantly higher than those of the sham group (*HI group vs. sham group $p<0.05$, \#HI group vs.HI + NGR1 group $p<0.05, \& \mathrm{HI}+\mathrm{NGR} 1$ group vs. $\mathrm{HI}+\mathrm{NGR} 1+\mathrm{ICI} 182780$ group

182780. The latencies of the HI + NGR $1+$ ICI 182780 group $(44.46 \pm 13.33,33.78 \pm 15.45,34.54 \pm 11.54$, and $35.54 \pm 15.31 \mathrm{~s}$, respectively) were significantly higher than those of the NGR1 treatment group $(p<0.05)$. The same results were found in the percentage of time spent in the target quadrant $(27.88 \pm 9.61 \%, p<0.05$ vs. the HI + NGR1 group) and the frequency $(1.03 \pm 1.11$, $p<0.01$ vs. the HI + NGR1 group) of crossing the target platform. The results suggested that NGR1 might exert its protective effects by targeting ERs.

\section{NGR1 Increased Activity of the PI3K-Akt-mTOR Signal Pathway via Estrogen Receptors}

\section{In Vitro and In Vivo}

$\mathrm{PI} 3 \mathrm{~K}$ is an intracellular phosphatidylinositol kinase that plays a major role in cerebral hypoxic-ischemic injury by regulating its downstream signaling pathway. Western blot analysis was used to detect expression levels of PI3K at $\mathrm{p}<0.05)(\mathbf{b}-\mathbf{f})$. The percentage of time spent in the target quadrant $\mathbf{g}$ and the frequency of crossing the target platform $\mathbf{h}$ were significantly higher in the sham group than those in the HI group; NGR1 treatment could increased the percentage of time and the frequency compared to the HI group. However, the protective effects could be reversed by ICI $182780(\mathbf{b}, \mathbf{g}-\mathbf{h})$. Data are expressed as mean \pm SEM. Sham $\mathrm{n}=8$, HI $\mathrm{n}=9$, HI+NGR1 $\mathrm{n}=9$, HI + NGR1+ICI $182780 \mathrm{n}=9$, $\mathrm{HI}+\mathrm{DMSO} \mathrm{n}=9 .{ }^{*} p<0.05 ; * * p<0.01 ; * * * p<0.001$

different times after hypoxic-ischemic injury in vitro (primary cortical neurons) and in vivo (ipsilateral hemisphere). As shown in Fig. 4a, expression of PI3K (1.54 \pm 0.60 in the control group) was significantly decreased at $12(0.88 \pm 0.42$, $p<0.05$ vs. the control group), $24(0.35 \pm 0.31, p<0.01$ vs. the control group), and $48 \mathrm{~h}(0.42 \pm 0.47, p<0.01 \mathrm{vs.}$ the control group) of reoxygenation cortical neurons. In vivo, expression of PI3K in the ipsilateral hemisphere was significantly decreased at $24(0.51 \pm 0.34$ vs. $1.32 \pm 0.78, p<0.05)$ and $48 \mathrm{~h}(0.30 \pm 0.32$ vs. $1.12 \pm 0.69, p<0.05)$ post HI compared with the contralateral hemisphere (Fig. 4b).

Akt is an important downstream target in the PI3K signal transduction pathway which can promote cell survival, inhibit apoptosis and maintain normal function as a key information molecule. As one of the important substrates for Akt, mTOR plays an important role in cell survival and differentiation. Among its downstream target proteins, 4EBP1 and p70S6k are the key signaling molecules, involved in cell-cycle regulation and promoting the synthesis of ribosomal translation regulatory proteins. 
Fig. 4 Expression of PI3K during OGD/R and HIBD. Representative Western blots for $\mathrm{PI} 3 \mathrm{~K}$ in primary cortical neurons and in $\mathrm{HI}$ rats. a PI3K was expressed at low levels 12, 24, $48 \mathrm{~h}$ after OGD/R. b Compared with the contralateral hemisphere, PI3K was expressed at low levels in the ipsilateral hemisphere 24 and $48 \mathrm{~h}$ after HI. $(* p<0.05 ; * * p<0.01$ compared with control/sham groups, $\mathrm{n}=5$, mean \pm SEM)
A
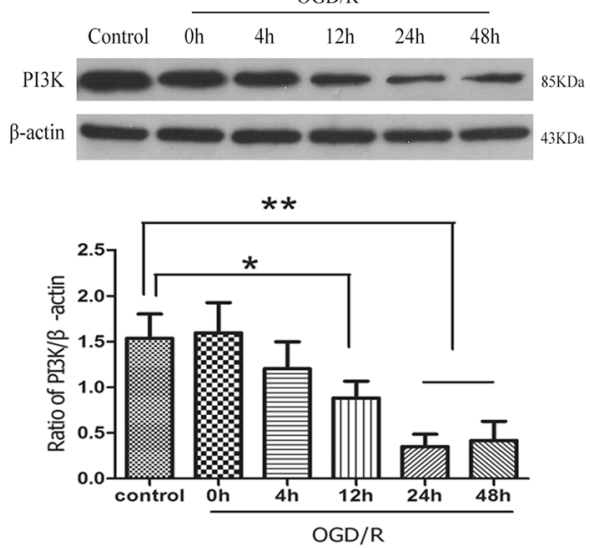

B
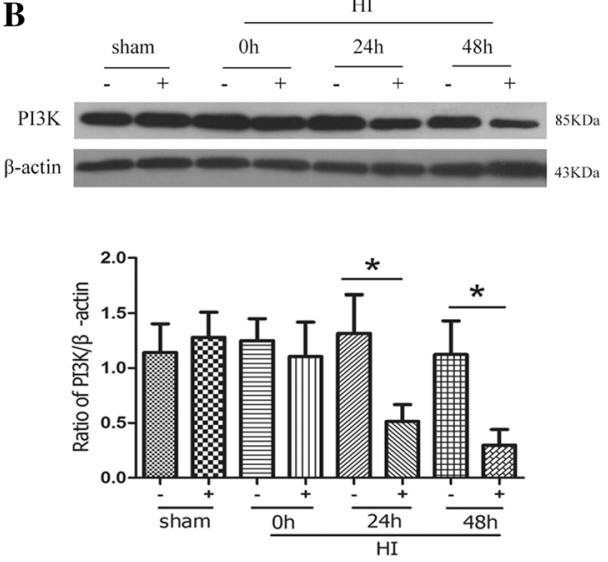

To detect activity of PI3K/Akt/mTOR signal path, primary neurons or hemisphere tissue were harvested at $24 \mathrm{~h}$ after OGD/R or HI injury for western blots. As seen in Fig. 5, the OGD/R group showed significant decrease in PI3K $(0.34 \pm 0.07$ vs. $1.09 \pm 0.46 p<0.01)$, phospho-Akt $(0.21 \pm 0.10$ vs. $0.86 \pm 0.42, p<0.01)$, phospho-mTOR $(0.46 \pm 0.21$ vs. $2.58 \pm 1.28, p<0.001)$, phospho-4EBP 1 $(0.24 \pm 0.09$ vs. $1.00 \pm 0.40, p<0.01)$, and phospho-p70S6k
$(0.57 \pm 0.33$ vs. $1.63 \pm 0.53, p<0.01)$ compared with the control group. Treatment with NGR1 $(10 \mu \mathrm{mol} / \mathrm{l})$ increased the expression levels of PI3K $(1.06 \pm 0.40, p<0.01$ vs. the OGD/R group), phospho-Akt $(0.88 \pm 0.46, p<0.01$ vs. the OGD/R group), phospho-mTOR $(1.83 \pm 0.43, p<0.01$ vs. the OGD/R group), phospho-4EBP1 $(1.05 \pm 0.54, p<0.01$ vs. the OGD/R group), and phospho-p70S6k $(1.55 \pm 0.83$, $p<0.05$ vs. the OGD/R group). However, pretreatment

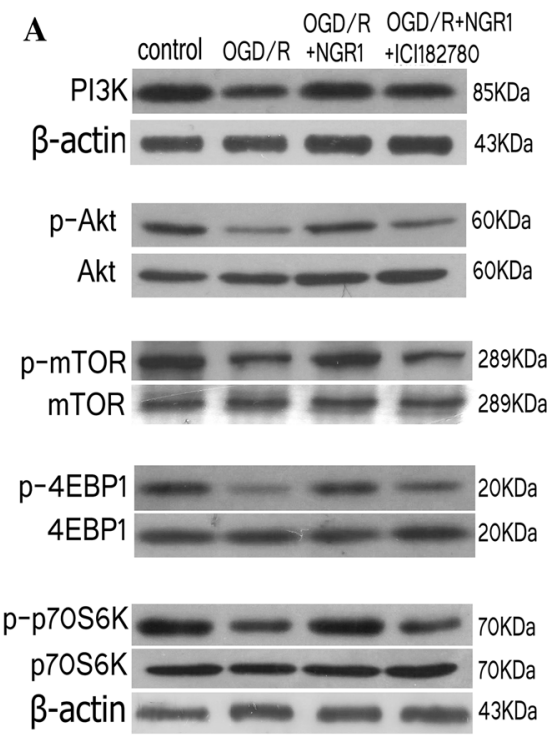

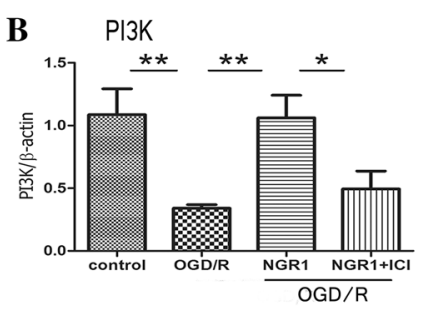
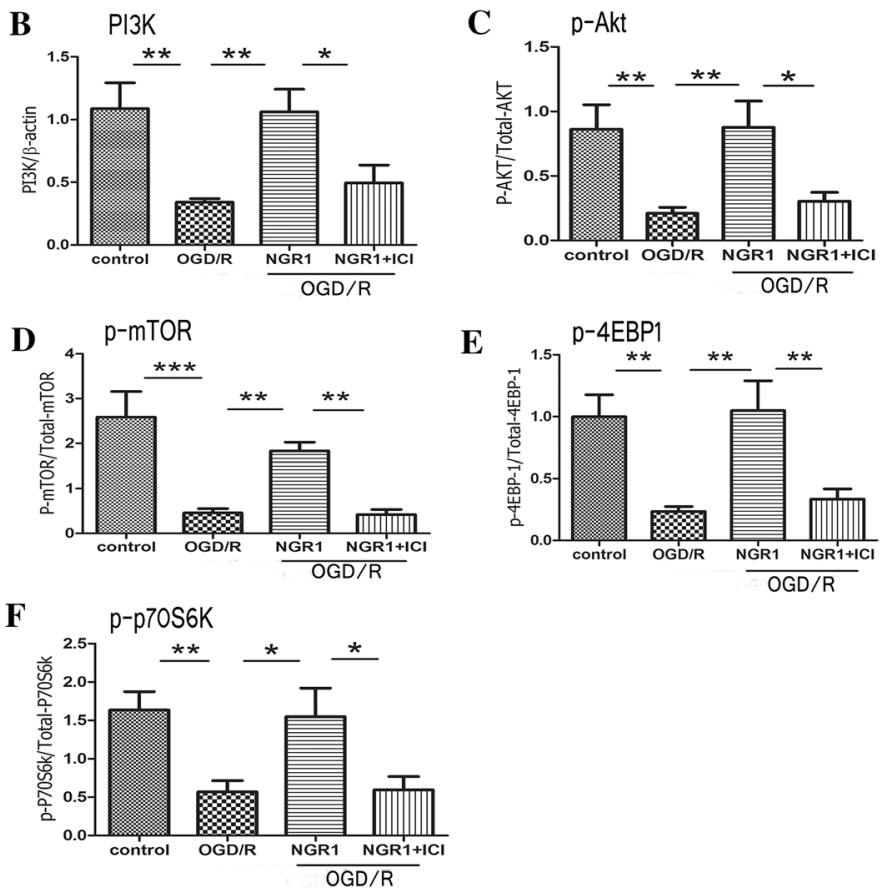

Fig. 5 Effects of NGR1 and ICI 182780 on PI3K-Akt-mTOR4EBP-1/p70S6K expression $24 \mathrm{~h}$ after OGD/R. Representative Western blots a for PI3K, phospho-Akt/Akt, phospho-mTOR/mTOR, phospho-p70S6K/p70S6K, and phospho-4EBP-1/4EBP-1 in primary cortical neurons. Western blot results showed that the expression of PI3K (b), phospho-Akt (c), phospho-mTOR (d), phospho-
p70S6K (e), and phospho-4EBP1 (f) was reduced in the OGD/R group compared with the control group. NGR1 (10 mmol/l) enhanced the expression of PI3K phospho-Akt, phospho-mTOR, phosphop70S6K, and phospho-4EBP1 in vitro. Pretreatment with ICI 182780 before NGR1 treatment could block the promoting effect. ${ }^{*} p<0.05$; $* * p<0.01 ; * * * p<0.001 ; \mathrm{n}=5$, mean \pm SEM 
with ICI 182780 before NGR 1 treatment in vitro resulted in the down-regulation of $\mathrm{PI} 3 \mathrm{~K}(0.49 \pm 0.32, p<0.05$ vs. the OGD/R + NGR1 group), phospho-Akt $(0.30 \pm 0.15$, $p<0.05$ vs. the OGD/R + NGR1 group), phospho-mTOR $(0.42 \pm 0.25, p<0.01$ vs. the OGD/R + NGR1 group), phospho-4EBP1 $(0.33 \pm 0.18, p<0.01$ vs. the OGD/R + NGR1 group), and phospho-p70S6k $(0.60 \pm 0.39, p<0.05$ vs. the OGD/R + NGR1 group) protein expression compared with the OGD + NGR1 group. As shown in Fig. 6, there was significant decrease in PI3K $(0.36 \pm 0.16$ vs. $1.00 \pm 0.35, p<0.01)$, phospho-Akt $(0.18 \pm 0.09$ vs. $0.52 \pm 0.15, p<0.01)$, phospho-mTOR $(0.79 \pm 0.22$ vs. $1.92 \pm 0.82, p<0.01)$, phospho-4EBP1 $(0.21 \pm 0.18$ vs. $0.96 \pm 0.34, p<0.01)$, and phospho-p70S6k $(0.76 \pm 0.49$ vs. $2.40 \pm 1.00, p<0.01)$ compared with the sham group. Treatment with NGR1 $(15 \mathrm{mg} / \mathrm{kg})$ increased the expression levels of PI3K $(0.98 \pm 0.42, p<0.01$ vs. the HI group), phospho-Akt $(0.41 \pm 0.05, p<0.05$ vs.the HI group), phospho-mTOR $(1.5 \pm 0.41, p<0.05$ vs. the HI group), phospho-4EBP1 $(0.70 \pm 0.30, p<0.05$ vs. the HI group), and phospho-p70S6k $(1.81 \pm 0.29, p<0.05$ vs. the HI group $)$. However, pretreatment with ICI 182780 before NGR 1 treatment in vivo resulted in the down-regulation of PI3K $(0.37 \pm 0.09, p<0.01$ vs. the HI + NGR1 group), phosphoAkt $(0.19 \pm 0.17, p<0.05$ vs. the HI + NGR1 group), phospho-mTOR $(0.82 \pm 0.16, p<0.05$ vs.the HI + NGR1 group $)$,
phospho-4EBP1 $(0.22 \pm 0.14, p<0.05$ vs. the HI + NGR 1 group), and phospho-p70S6k ( $0.85 \pm 0.47, p<0.05$ vs. the $\mathrm{HI}+\mathrm{NGR} 1$ group) protein expression compared with the $\mathrm{HI}+\mathrm{NGR} 1$ group. The results indicated that NGR1 might regulate the PI3K-Akt-mTOR signal pathway via ERs in hypoxic-ischemic brain injury.

\section{NGR1 Downregulated JNK Signal Pathway via Estrogen Receptors in Vitro and in Vivo}

The phosphorylation of JNK and c-JUN were examined $24 \mathrm{~h}$ after OGD/R or HI injury. Western blot analysis showed that OGD/R injury resulted in remarkably increased expression of both phospho-JNK $(1.38 \pm 0.56$ vs. $0.34 \pm 0.14$, $p<0.01)$ and phospho-c-JUN $(1.56 \pm 0.63$ vs. $0.31 \pm 0.24$, $p<0.01)$ in primary cortical neurons compared with the control group. NGR 1 treatment significantly decreased the expression levels of phospho-JNK $(0.63 \pm 0.33, p<0.01$ vs. the OGD/R group) and phospho-c-JUN $(0.72 \pm 0.57$, $p<0.05$ vs. the OGD/R group), and the effects of NGR1 were blocked by ICI 182780 . Pretreatment with ICI 182780 before NGR1 treatment led to higher levels of phospho-JNK $(1.18 \pm 0.36)$ and phospho-c-JUN $(1.65 \pm 0.40)$ than those in OGD/R + NGR1 group $(p<0.05)($ Fig. $7 \mathrm{a}-\mathrm{c})$.

Similarly, in the HI group, the expression of phospho-JNK $(0.99 \pm 0.44$ vs. $0.28 \pm 0.20, p<0.001)$ and
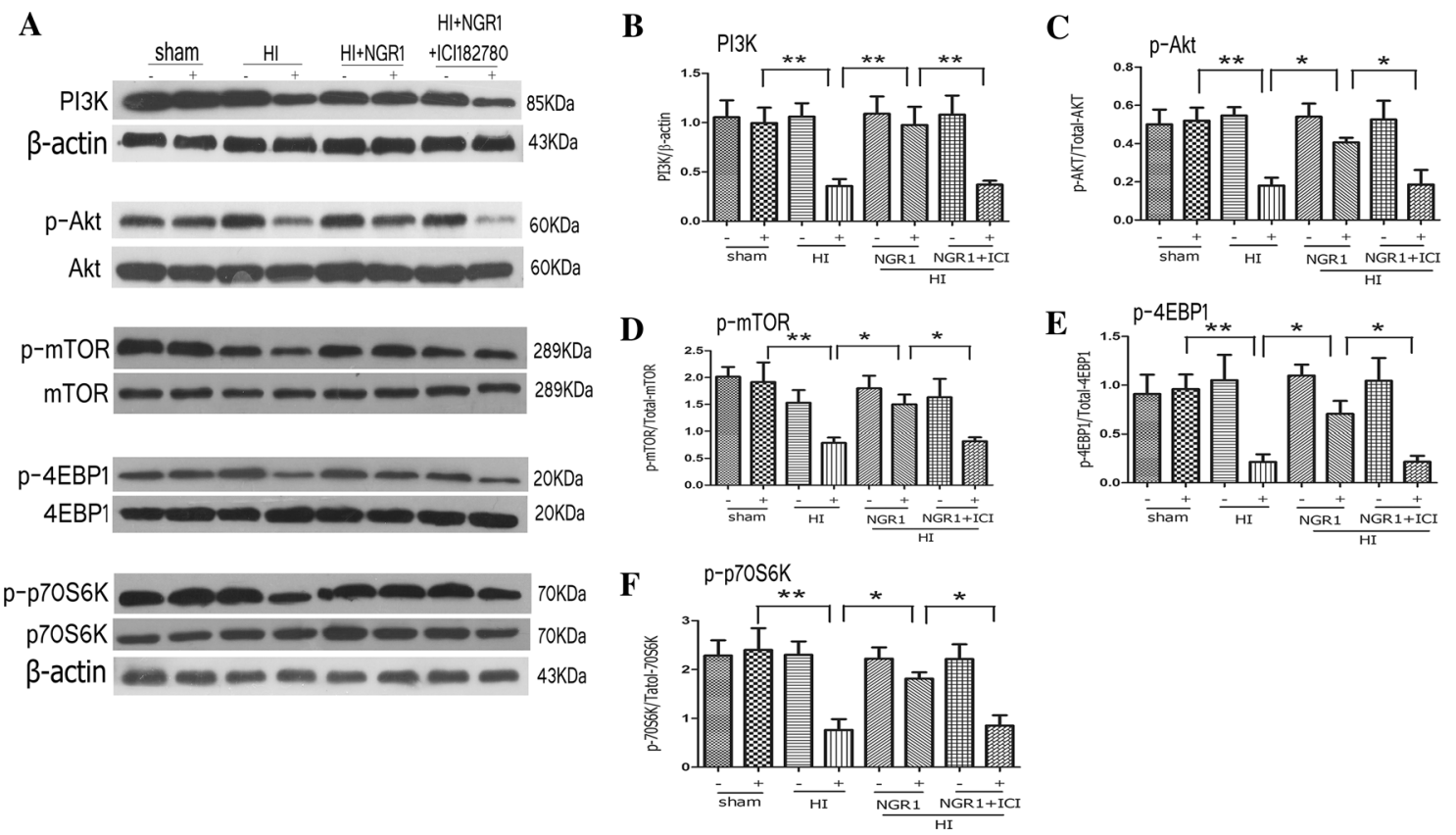

Fig. 6 Effects of NGR1 and ICI 182780 on PI3K-Akt-mTOR4EBP-1/P70S6K expression $24 \mathrm{~h}$ after HI. Representative Western blots a for PI3K, phospho-Akt/Akt, phospho-mTOR/mTOR, phospho-P70S6K/P70S6K, and phospho-4EBP-1/4EBP-1 in vivo. Western blot results showed that the expression of PI3K (b), phospho-Akt (c), phospho-mTOR (d), phospho-p70S6K (e), and phospho-4EBP1 (f) was significantly decreased in the HI group compared with the sham group. NGR1 $(15 \mathrm{mg} / \mathrm{kg})$ enhanced the expression of PI3K phospho-Akt, phospho-mTOR, phospho-p70S6K, and phospho4EBP1 in vivo. Pretreatment with ICI 182780 before NGR1 treatment could block the promoting effects. ${ }^{*} p<0.05 ; * * p<0.01 ; \mathrm{n}=5$, mean \pm SEM 


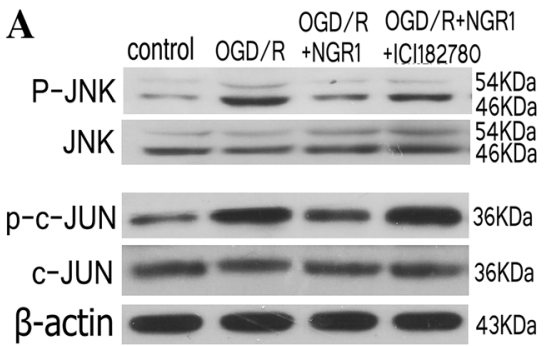

D

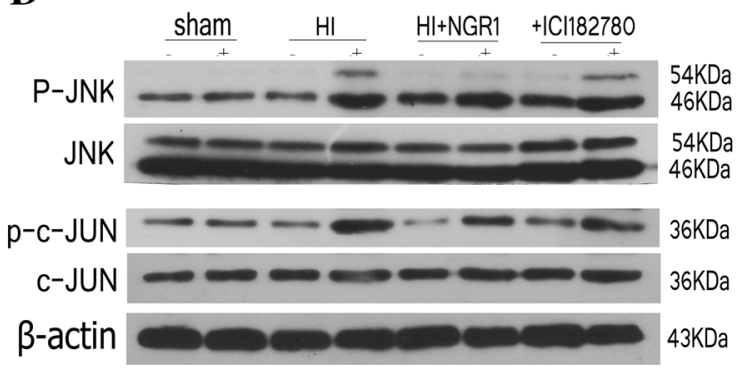

Fig. 7 Effects of NGR1 and ICI 182780 on JNK-c-JUN expression $24 \mathrm{~h}$ after OGD/R and HI. Representative Western blots for phosphoJNK/JNK and phospho-c-JUN/c-JUN in primary cortical neurons (a) and for phospho-JNK/JNK and phospho-c-JUN/c-JUN in vivo (d). Western blot analysis showed that compared with the OGD/R or HI

phospho-c-JUN $(1.26 \pm 0.56$ vs. $0.41 \pm 0.22, p<0.001)$ increased in the ipsilateral hemisphere compared with the sham group, and NGR1 attenuated the activation of phospho-JNK $(0.47 \pm 0.28, p<0.01$ vs. the HI group $)$ and phospho-c-Jun $(0.70 \pm 0.24, p<0.05$ vs. the HI group). Pretreatment with ICI 182780 before NGR1 treatment led to higher levels of phospho-JNK $(0.94 \pm 0.25, p<0.01$ vs. the HI+ NGR1 group) and phospho-c-JUN $(1.19 \pm 0.30 p<0.05$ vs. the HI + NGR1 group) than those in the NGR1 group (Fig. 7d-f).

The results indicated that NGR1 might inhibit the activity of JNK/c-JUN signal pathway by acting ERs and reduced the neuronal apoptosis.

\section{NGR1 Exerted Neuroprotective Effects via Estrogen Receptors and PI3K}

The preceding results showed that NGR1 could exert neuroprotective effects by regulating the PI3K-Akt-mTOR/ JNK signal pathways, but these effects could be reversed by blocking the ERs. Previous research [60-64] showed that PI3K could interact with ERs. To further explore the relationship between NGR1, PI3K and ERs, LY294002 (PI3K inhibitor) and 740Y-P (PI3K agonist) were used.

As shown in Fig. 8, with a optimum concentration of LY294002 treatment $(20 \mu \mathrm{mol} / \mathrm{l})$ [40] (Fig. 8a), the OGD + NGR1 + LY294002 group showed lower cell viability
C

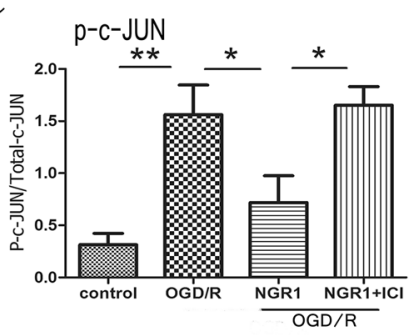

$\mathbf{F}$

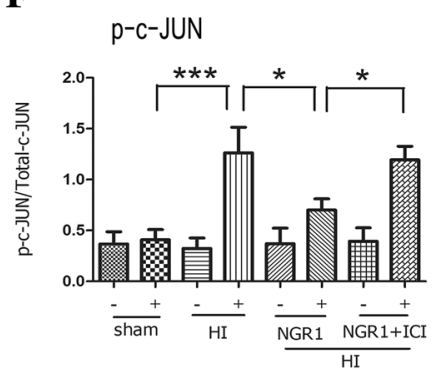

group, NGR1 inhibited the expression of phospho-JNK and phosphoc-JUN in vitro (b, c) and in vivo (e, f). Pretreatment with ICI 182780 before NGR1 treatment could block the inhibiting effects of NGR1. $* p<0.05 ; * * p<0.01 ; \mathrm{n}=5$, mean \pm SEM

$(46.99 \pm 17.50$ vs. $75.53 \pm 18.94 \%, p<0.05)$ and more LDH leakage $(39.40 \pm 7.40$ vs. $28.18 \pm 6.40 \%, p<0.05)$ than the NGR1 treatment group, which suggested that the neuroprotective effects of NGR1 were inhibited (Fig. 8c, d). At the same time, the phosphorylation of Akt $(0.18 \pm 0.12 \mathrm{vs}$. $0.46 \pm 0.18, p<0.05)$ and mTOR $(0.31 \pm 0.16$ vs. $0.88 \pm 0.28$, $p<0.01)$ was lower in the OGD/R + NGR $1+\mathrm{LY} 294002$ group than that in the OGD/R + NGR1 group, while the phosphorylation of JNK $(0.96 \pm 0.32$ vs. $0.49 \pm 0.17$, $p<0.05)$ was higher than that in the OGD/R + NGR1 group (Fig. 8e-h). To further explore the role of ERs in the PI3K signal pathway, the optimal concentration of 740Y-P was tested and found to be $20 \mu \mathrm{mol} / \mathrm{l}$ (Fig. $8 \mathrm{~b}$ ); this concentration was used in the following investigation. The results showed that ICI 182780 could reverse the neuroprotective effects of NGR1 and aggravate neural injury. However, when 740Y-P was used in the OGD/R + NGR $1+$ ICI 182780 group, the expression of phospho-Akt $(0.46 \pm 0.17$ vs. $0.16 \pm 0.11, p<0.01)$ and phospho-mTOR $(0.99 \pm 0.39$ vs. $0.35 \pm 0.23, p<0.01)$ was activated and the expression of phospho-JNK $(0.18 \pm 0.17$ vs. $1.28 \pm 0.50, p<0.001)$ was inhibited compared with the OGD/R group (Fig. 8e-h). Simultaneously, the results showed higher cell viability $(69.70 \pm 17.52$ vs. $47.34 \pm 21.36 \%, p<0.05)$ and less LDH leakage $(24.27 \pm 9.30$ vs. $38.97 \pm 10.20 \%, p<0.05)$ in the OGD/R + NGR1 + ICI $182780+740$ Y-P group compared with the OGD/R + NGR1 + ICI 182780 group(Fig. 8c, d). 
$\mathbf{A}$

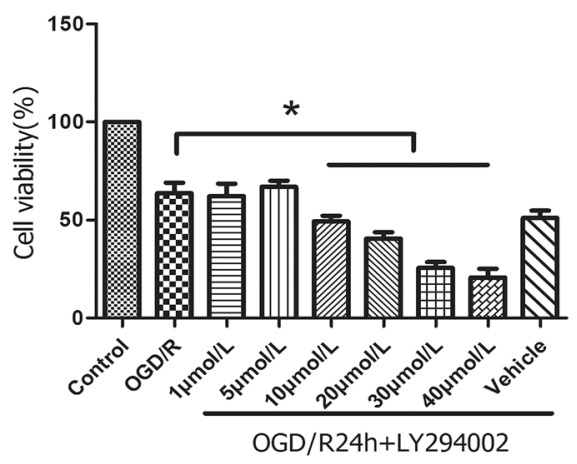

$\mathbf{C}$

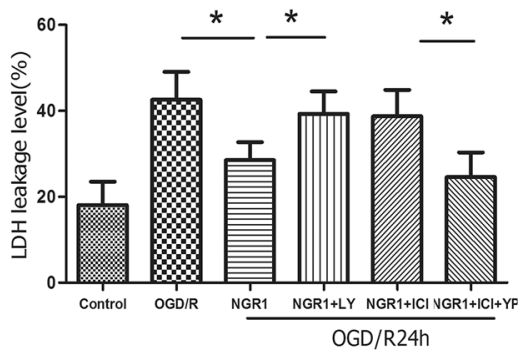

B

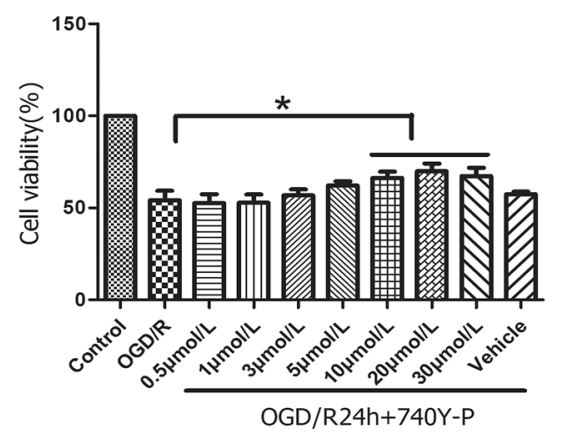

D

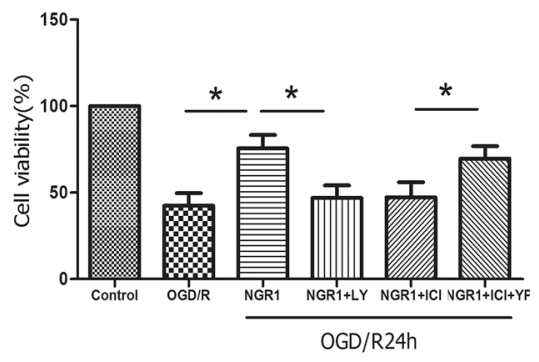

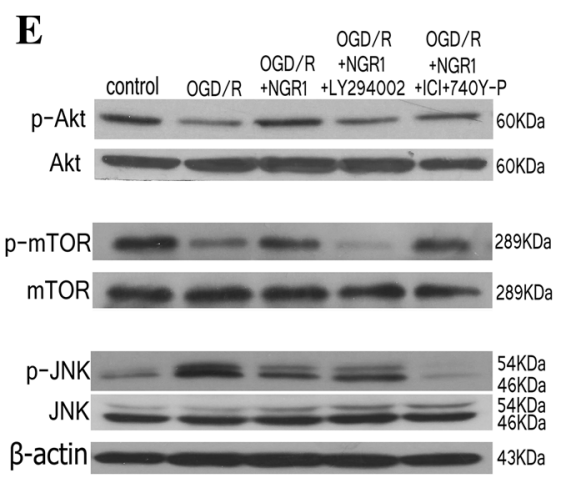

F

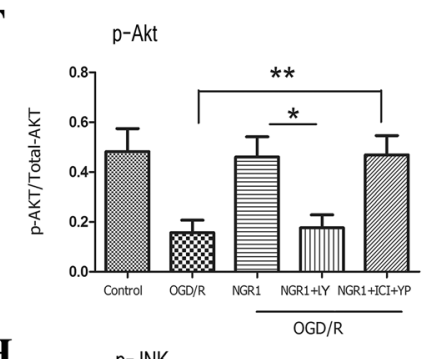

$\mathbf{H}$

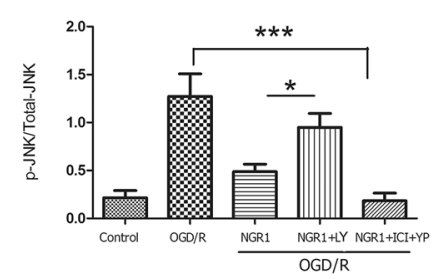

G $\quad p-m T O R$

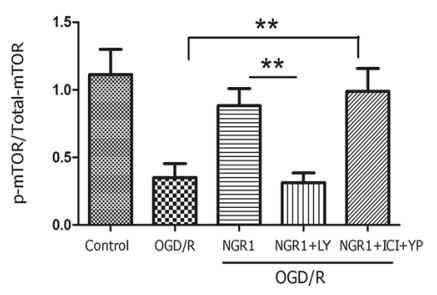

Fig. 8 Effects of LY294002/740Y-P during OGD/R. a The optimal concentration of LY294002 was $20 \mu \mathrm{mol} / \mathrm{l}$. b The optimal concentration of 740Y-P was $20 \mu \mathrm{mol} / \mathrm{l}$. LY294002 treatment could accelerate LDH leakage $\mathbf{c}$ and reduce cell viability $\mathbf{d}$ in the OGD/R + NGR1 + LY294002 group compared to the NGR1 treatment group. 740Y-P treatment could promote cell viability and inhibit LDH leakage in the OGD/R + NGR1 + ICI 182780+740Y-P group compared to the $\mathrm{OGD} / \mathrm{R}+\mathrm{NGR} 1+\mathrm{ICI} 182780$ group. $(* p<0.05$, $\mathrm{n}=6$, mean $\pm \mathrm{SEM}$ ). Representative Western blots e for phospho-

These results indicated that ERs might regulate the activation of Akt-mTOR/JNK through interaction with PI3K, and NGR1 might cause PI3K activation to decrease cell damage after OGD/R by targeting ERs.
Akt/Akt (f), phospho-mTOR/mTOR (g) and phospho-JNK/JNK (h) in primary cortical neurons. In the OGD/R + NGR1 + LY294002 group, the phosphorylation of Akt and mTOR was lower than that in the OGD/R + NGR1 group, with a higher phosphorylation of JNK than that in the OGD/R + NGR1 group; In the OGD/R + NGR1 + ICI $182780+740$ Y-P group, Akt/mTOR phosphorylation was higher and JNK phosphorylation was lower than that in the OGD/R group. $* p<0.05$; ** $p<0.01 ; * * * p<0.001 ; \mathrm{n}=5$, mean \pm SEM

\section{Discussion}

HIE is a common neurologic disease in newborns, but there is currently a lack of promising therapy [3]. Many studies have shown that estrogen provides neuroprotective effects in experimental cerebral ischemia $[20,21]$. These protective effects are mediated by ligand interactions with two 
primary classical ERs, ER $\alpha$ and ER $\beta$ [65]. Research has shown that the distribution patterns of ER $\alpha$ and ER $\beta$ are similar in male and female brains. Especially in the cortical and hippocampal regions [66], sex differences were found to be absent [67]. However, studies suggested that estrogen exhibited universal protection against experimental ischemia injury via ERs in female but not male brains [68]. The differences may be due at least in part to the fact that circulating estrogens have free access to all brain regions. As a phytoestrogen, NGR1 has been found to exhibit a number of treatment effects and exert direct anti-inflammatory and antiapoptotic effects on cardiomyocytes [26], vascular endothelial cells [69], podocytes [70], and neurons [18, 44] through acting ERs. Some scholars reported that NGR1 treatment significantly improved cognitive function in the APP/PS1 double-transgenic mouse model of Alzheimer's disease [71]. One study demonstrated neuroprotective effects of NGR 1 in an adult rat model of cerebral ischemia/reperfusion [18]. However, research has revealed that the immature brain responded differently to treatment than the mature brain in laboratory animals [3]. In fact, therapies designed to ameliorate brain injury in adults may worsen outcomes in neonates [72]. Hence, effective therapies for neonatal HIE need to be explored. Although some preliminary experimental results are available [19], whether NGR1 exerts short-term or long-term protective effects and the underlying mechanisms are largely unknown. Therefore, the evaluation of the early effects and long-term therapeutic effects of NGR1 is of great clinical significance.

In the present study, a series of experiments were designed to explore the neuroprotective effects and underlying mechanisms of NGR1 in a neonatal hypoxic-ischemic injury model. The pivotal findings are as follows. (1) NGR1 significantly attenuated neuronal injury in the neonatal $\mathrm{HI}$ model in vitro and in vivo. Most importantly, NGR1 had contributed to the long-term recovery of neurological function in the HI rats. (2) NGR1 exerted neuroprotective effects through regulating the PI3K-Akt-mTOR/JNK signal pathways by targeting ERs.

HIE [11] can develop as a result of circulatory and energy metabolism disorders, leading to a series of pathophysiological processes, including oxidative stress, mitochondrial impairment, apoptosis, and necroptosis. These injuries in the developing brain often lead to lasting neurological impairments, such as cerebral palsy, epilepsy, mental retardation, and learning and memory disorders. Therefore, reducing neuronal death and promoting neuronal survival and proliferation are important strategies for reducing the occurrence of long-term neurological sequelae [26]. Our results indicated that NGR1 possessed protective effects both in vitro and in vivo. NGR1 was observed significantly to improve neuronal cell viability and reduce the LDH leakage rate 4 and $24 \mathrm{~h}$ after OGD/R (Fig. 1). The inhibition of cortical neuronal apoptosis was observed $24 \mathrm{~h}$ after $\mathrm{HI}$ injury and the decrease of infarct volume was examined $48 \mathrm{~h}$ after $\mathrm{HI}$ injury in HI+NGR1 group (Fig. 2). These findings are consistent with a recent study in an adult cerebral ischemia-reperfusion brain injury model, which found that NGR1 therapy reduced brain damage after ischemia [18]. However, that study used a higher concentration of NGR $1(25 \mathrm{mmol} / \mathrm{l}$ in vitro and $20 \mathrm{mg} / \mathrm{kg}$ in vivo) than our study $(10 \mathrm{mmol} / \mathrm{l}$ in vitro and $15 \mathrm{mg} / \mathrm{kg}$ in vivo). There may be two reasons for the difference. (1) We used cells from different culture days and rats of different ages. (2) NGR1 was administered after OGD/R or $\mathrm{HI}$ in our study, not as a pretreatment. Importantly, our results indicated that NGR1 contributed to the long-term recovery of neurological function in the neonatal $\mathrm{HI}$ model in addition to reducing apoptosis. NGR1 treatment reduced brain atrophy 6 weeks after HI injury (Fig. 2). Moreover, the results of beam walking ( 5 weeks after HIE) and the water maze test (5-6 weeks after HIE) showed that NGR1 significantly restored limb coordination and improved learning and memory in the impaired rats (Fig. 3).

Hypoxic-ischemic brain injury directly results in a large amount of neuronal death. Therefore, reducing neuronal death and promoting neuronal survival and proliferation are important strategies for reducing the occurrence of long-term neurological sequelae [26]. Apoptosis is reported to be responsible for a significant proportion of the HI-induced neuronal loss [72], and multiple apoptosisrelated signal pathways, such as PI3K-Akt-mTOR/JNK, are involved in neuronal death after stroke [34, 40, 41]. Our results showed significant inhibition of the PI3K-AktmTOR-4EBP1/p70S6k signal pathway at $24 \mathrm{~h}$ following OGD/R or HI injury (Figs. 5, 6). At the same time, JNKanother important signaling protein downstream of Akt, which can be inhibited by Akt directly or indirectly-was significantly activated. These results suggested that neuronal apoptosis might be related to the inhibition of PI3KAkt-mTOR and the activity of JNK-c-JUN during HIBD. Some other researchers [44, 49-51, 73, 74] have found similar results indicating that cerebral ischemia induced the robust activation of JNK signaling and inhibition of PI3K-Akt-mTOR pathway activity. NGR1 treatment could increase the expression of PI3K, phospho-Akt, and phospho-mTOR (Figs. 5, 6) and reduce the activity of the JNK signaling pathway $24 \mathrm{~h}$ after OGD/R or HI brain injury (Fig. 7). These results indicated that NGR1 could likely reduce neuronal apoptosis by regulating the activity of the PI3K-Akt-mTOR/JNK signal pathways. NGR1 treatment could improve the cell survival rate in vitro and reduce infarct volume and promote long-term neurobehavioral recovery and improvement in vivo by inhibiting neuronal apoptosis. Previous studies showed that mTOR accelerated angiogenesis [75] and neuronal regeneration [76] in many neurologic injuries in addition to reducing neuronal 
apoptosis. Perhaps the long-term protective effects of NGR1 were also related to its activation of mTOR and promotion of neuroregeneration.

We further explored whether NGR1 achieved its neuroprotective effects via ERs. As a predominant phytoestrogen extracted from $P$. notoginseng, NGR1 was previously found to perform its function through acting ERs [13, 15, 18, 19]. Mounting evidence showed that ER $\alpha$ and ER $\beta$ expression was reduced during neuronal ischemia $[19,77]$. Kraczkowski [78] indicated that the downregulation of ERs might be related to the ontogenesis of brain $\mu$-opioid receptors during HIBD. As an ERs agonist, NGR 1 may act on ER $\alpha / \beta$ and improve the role of ERs during HIBD $[18,19]$. Our results indicated that pretreatment with ICI 182780 reduced the survival rate of cortical neurons in vivo and increased brain edema and cerebral infarction volume in vitro compared with the HI + NGR1 group. Moreover, the long-term protective effects of NGR1 were suppressed by ICI 182780 . These results suggested that NGR1 exerted its protective effects via ERs.

Studies on a variety of cells-such as endothelial cells [79], MCF-7 breast cancer cells [80], and neurons [81-83]have found that ERs can interact directly with PI3K or bind to the PI3K p85 subunit through scaffold proteins such as CAV-1, connective proteins such as Src and Shc, and growth factors, then activate the downstream Akt, causing a series of signal pathway cascades, such as the Akt-mTOR/JNK signal pathway [60-63]. Our results showed that pretreatment with ICI 182780 could inhibit the activity of PI3KAkt-mTOR and increase the activity of the JNK signal pathway. These results suggested that NGR1 regulated the PI3K-Akt-mTOR/JNK signal pathways via acting ERs. In order to further validate this finding, we used LY294002 (PI3K inhibitor) and 740Y-P (PI3K agonist) to perform related experiments. The results (Fig. 8) revealed that the protective effects of NGR1 were significantly inhibited after adding LY294002, the expression of phospho-Akt and phospho-mTOR decreased and that of JNK increased in the OGD/R + NGR1 + LY294002 group. However, 740Y-P could reverse the inhibition of NGR1's neuroprotective effects induced by ICI182780. Simultaneously, phosphoAkt expression increased and phospho-JNK expression decreased in the 740Y-P agonist group. These results suggested that NGR1 might exert a neuroprotective effects by targeting ERs and regulating PI3K.

In conclusion, the present study demonstrated that NGR1 inhibited neuronal apoptosis and promoted neuronal survival, exerting an important neuroprotective effects against HIBD in neonates through targeting ERs and regulating the PI3K-Akt-mTOR/JNK signal pathway. Our findings suggested that NGR1 might be a potent new therapeutic compound for neonatal hypoxia-ischemia brain damage treatment.
Acknowledgements This study was supported by the National Natural Science Foundation of China (81401234). We thank LetPub (http:// www.letpub.com) for its linguistic assistance during the preparation of this manuscript.

Open Access This article is distributed under the terms of the Creative Commons Attribution 4.0 International License (http://creativeco mmons.org/licenses/by/4.0/), which permits unrestricted use, distribution, and reproduction in any medium, provided you give appropriate credit to the original author(s) and the source, provide a link to the Creative Commons license, and indicate if changes were made.

\section{References}

1. Doycheva D, Shih G, Chen H, Applegate R, Zhang JH, Tang J (2013) Granulocyte-colony stimulating factor in combination with stem cell factor confers greater neuroprotection after hypoxicischemic brain damage in the neonatal rats than a solitary treatment. Transl Stroke Res 4:171-178

2. Shetty J (2015) Neonatal seizures in hypoxic-ischaemic encephalopathy-risks and benefits of anticonvulsant therapy. Dev Med Child Neurol 57:40-43

3. Volpe JJ (2001) Perinatal brain injury: from pathogenesis to neuroprotection. Dev Disabil Res Rev 7:56-64

4. Badr Zahr LK, Purdy I (2006) Brain injury in the infant: the old, the new, and the uncertain. J Perinat Neonatal Nurs 20:163-175

5. Gill MB, Perez-Polo JR (2008) Hypoxia ischemia-mediated cell death in neonatal rat brain. Neurochem Res 33:2379-2389

6. Northington FJ, Chavez-Valdez R, Martin L (2011) Neuronal cell death in neonatal hypoxia-ischemia. Ann Neurol 69:743-758

7. Shankaran S (2012) Hypoxic-ischemic encephalopathy and novel strategies for neuroprotection. Clin Perinat 39:919-929

8. Fathali N, Khatibi NH, Ostrowski RP, Zhang JH (2011) The evolving landscape of neuroinflammation after neonatal hypoxiaischemia. Acta Neurochir Suppl 111:93-100

9. Hara H, Fink K, Endres M, Friedlander RM, Gagliardini V, Moskowitz MA (1997) Attenuation of transient focal cerebral ischemic injury in transgenic mice expressing a mutant ICE inhibitory protein. J Cereb Blood Flow Metab 17:370-375

10. Malagelada C, XIfrò X, Mińano A, Sabrìa J, Rodriguez-Alvarez J (2005) Contribution of caspase-mediated apoptosis to the cell death caused by oxygen-glucose deprivation in cortical cell cultures. Neurobiol Dis 20:27-37

11. Dixon BJ, Reis C, Ho WM, Zhang JH, Tang J (2015) Neuroprotective strategies after neonatal hypoxic ischemic encephalopathy. Int J Mol Sci 16(9):22368-22401

12. Ge ZR, Xu MC, Huang YU, Zhang CJ, Lin JE, Ruan CW (2016) Cardioprotective effect of notoginsenoside R1 in a rabbit lung remote ischemic postconditioning model via activation of the TGF- $\beta 1 /$ TAK1 signaling pathway. Exp Ther Med 11(6):2341-2348

13. Sun B, Xiao J, Sun XB, Wu Y (2013) Notoginsenoside R1 attenuates cardiac dysfunction in endotoxemic mice: an insight intooestrogen receptor activation and PI3K/Akt signalling. Br J Pharmacol 168(7):1758-1770

14. Xia KP, Ca HM, Shao CZ (2015) Protective effect of notoginsenoside $\mathrm{R} 1$ in a rat model of myocardial ischemia reperfusion injury by regulation of Vitamin D3 upregulated protein 1/NF- $\mathrm{KB}$ pathway. Pharmazie 70(11):740-744

15. Zhong L, Zhou XL, Liu YS, Wang YM, Ma F, Guo BL, Yan ZQ, Zhang QY (2015) Estrogen receptor $\alpha$ mediates the effects of notoginsenoside R1 on endotoxin-induced inflammatory and 
apoptotic responses in H9c2 cardiomyocytes. Mol Med Rep 12(1):119-126

16. Zhao J, Shi Z, Liu S, Li J, Huang W (2014) Ginsenosides Rg1 from Panax ginseng: a potential therapy for acute liver failure patients? Evid Based Complement Alternat Med 2014:538059

17. Gui D, Wei L, Jian G, Guo Y, Yang J, Wang N (2014) Notoginsenoside R1 ameliorates podocyte adhesion under diabetic condition through $\alpha 3 \beta 1$ integrin upregulation in vitro and in vivo. Cell Physiol Biochem 34(6):1849-1862

18. Meng X, Wang M, Wang X, Sun G, Ye J, Xu H, Sun X (2014) Suppression of NADPH oxidase-and mitochondrion-derived superoxide by Notoginsenoside R1 protects against cerebral ischemia-reperfusion injury through estrogen receptor-dependent activation of Akt/Nrf2 pathways. Free Radic Res 48(7):823-838

19. Wang Y, Tu L, Li Y, Chen D, Wang S (2016) Notoginsenoside $\mathrm{R} 1$ protects against neonatal cerebral hypoxic-ischemic injury through estrogen receptor-dependent activation of endoplasmic reticulum stress pathways. J Pharmacol Exp Ther 357(3):591-605

20. Cue L, Diaz F, Briegel KJ, Patel HH, Raval AP (2015) Periodic estrogen receptor-beta activation: novel approach to prevent ischemic brain damage. Neurochem Res 40(10):2009-2017

21. Dubal DB, Zhu H, Yu J, Rau SW, Shughrue PJ, Merchenthaler I, Kindy MS, Wise PM (2001) Estrogen receptor alpha, not beta, is a critical link in estradiol mediated protection against brain injury. Proc Natl Acad Sci USA 98(4):1952-1957

22. Liu B, Zhang J, Liu W, Liu N, Fu X, Kwan H, Liu S, Liu B, Zhang S, Yu Z, Liu S (2016) Calycosin inhibits oxidative stress-induced cardiomyocyte apoptosis via activating estrogen receptor- $\alpha / \beta$. Bioorg Med Chem Lett 26(1):181-185

23. Hsu JT, Chen TH, Chiang KC, Kuo CJ, Lin CJ, Yeh TS (2015) Role of p38 MAPK pathway in 17 $\beta$-estradiol-mediated attenuation of hemorrhagic shock-induced hepatic injury. J Appl Physiol 118(2):187-192

24. Wang Y, Li X, Wang J, Shi H, Bi W, Hou W, Zhang X (2015) $\beta$-estradiol mediates upregulation of stromal cell-derived factor- 1 in the retina through activation of estrogen receptor in an ischemia-reperfusion injury model. Graefes Arch Clin Exp Ophthalmol 253(1):17-23

25. Miller NR, Jover T, Cohen HW, Zukin RS, Etgen AM (2005) Estrogen can act via estrogen receptor alpha and beta to protect hippocampal neurons against global ischemia-induced cell death. Endocrinology 146(7):3070-3079

26. Nijboer CH, van der Kooij MA, van Bel F, Ohl F, Heijnen CJ, Kavelaars A (2010) Inhibition of the jnk/ap-1 pathway reduces neuronal death and improves behavioral outcome after neonatal hypoxic-ischemic brain injury. Brain Behav Immun 24(5):812-821

27. Liu M, Bamodu OA, Huang WC, Zucha MA, Lin YK, Wu ATH, Huang CC, Lee WH, Yuan CC, Hsiao M, Deng L, Tzeng YM, Yeh CT (2017) 4-Acetylantroquinonol B suppresses autophagic flux and improves cisplatin sensitivity in highly aggressive epithelial cancer through the PI3K/Akt/mTOR/p70S6K signaling pathway. Toxicol Appl Pharmacol 325:48-60

28. Fruman DA, Meyers RE, Cantley LC (1998) Phosphoinositide kinases. Annu Rev Biochem 67:481-507

29. Hennessy BT, Smith DL, Ram PT et al (2005) Exploiting the PI3K/AKT pathway for cancer drug discovery. Nat Rev Drug Discov 4(12):988-1004

30. Ouyang YB, Tan Y, Comb M, Liu CL, Martone ME, Siesjö BK, Hu BR (1999) Survival- and death-promoting events after transient cerebral ischemia: phosphorylation of Akt, release of cytochrome $\mathrm{C}$ and activation of caspase-like proteases. J Cereb Blood Flow Metab 19(10):1126-1135

31. Castaneda CA, Cortes-Funes H, Gomez HL, Ciruelos EM (2010) The phosphatidyl inositol 3-kinase/AKT signaling pathway in breast cancer. Cancer Metastasis Rev 29(4):751-759
32. Wullschleger S, Loewith R, Hall MN (2006) TOR signaling in growth and metabolism. Cell 124(3):471-484

33. Martelli AM, Tazzari PL, Evangelisti C, Chiarini F, Blalock WL, Billi AM, Manzoli L, McCubrey JA, Cocco L (2007) Targeting the phosphatidylinositol 3-kinase/Akt/mammalian target of rapamycin module for acute myelogenous leukemia therapy: from bench to bedside. Curr Med Chem 14(19):2009-2023

34. Kamada H, Nito C, Endo H, Chan PH (2007) Bad as a converging signaling molecule between survival PI3-K/Akt and death JNK in neurons after transient focal cerebral ischemia in rats. J Cereb Blood Flow Metab 27(3):521-533

35. Ge XH, Zhu GJ, Geng DQ, Zhang HZ, He JM, Guo AZ, Ma LL, $\mathrm{Yu}$ DH (2017) Metformin protects the brain against ischemia/ reperfusion injury through PI3K/Akt1/JNK3 signaling pathways in rats. Physiol Behav 170:115-123

36. Pan J, Pei DS, Yin XH, Hui L, Zhang GY (2006) Involvement of oxidative stress in the rapid Akt1 regulating a JNK scaffold during ischemia in rat hippocampus. Neurosci Lett 392(1-2):47-51

37. Zhang QG, Wu DN, Han D, Zhang GY (2007) Critical role of PTEN in the coupling between PI3K/Akt and JNK1/2 signaling in ischemic brain injury. FEBS Lett 581(3):495-505

38. Zhang M, Yan H, Li S, Yang J (2017) Rosmarinic acid protects rat hippocampal neurons from cerebral ischemia/reperfusion injury via the Akt/JNK3/caspase-3 signaling pathway. Brain Res 1657:9-15

39. Zhao H, Sapolsky RM, Steinberg GK (2006) Phosphoinositide3-kinase/akt survival signal pathways are implicated in neuronal survival after stroke. Mol Neurobiol 34(3):249-270

40. Xu XH, Li GL, Wang BA, Qin Y, Bai SR, Rong J, Deng T, Li Q (2015) Diallyl trisufide protects against oxygen glucose deprivation-induced apoptosis by scavenging free radicals via the PI3K/ Akt -mediated Nrf2/HO-1 signaling pathway in B35 neural cells. Brain Res 1614:38-50

41. Zhao H, Mitchell S, Koumpa S, Cui YT, Lian Q, Hagberg H, Johnson MR, Takata M, Ma D (2016) Heme oxygenase-1 mediates neuroprotection conferred by argon in combination with hypothermia in neonatal hypoxia-ischemia brain injury. Anesthesiology 125(1):180-192

42. Endo H, Nito C, Kamada H, Yu F, Chan PH (2006) Akt/GSK3beta survival signaling is involved in acute brain injury after subarachnoid hemorrhage in rats. Stroke 37(8):2140-2146

43. Hong Y, Shao A, Wang J, Chen S, Wu H, McBride DW, Wu Q, Sun X, Zhang J (2014) Neuroprotective effect of hydrogen-rich saline against neurologic damage and apoptosis in early brain injury following subarachnoid hemorrhage: possible role of the Akt/GSK3 $\beta$ signaling pathway. PLoS ONE 9(4):e96212

44. Noshita N, Lewén A, Sugawara T, Chan PH (2001) Evidence of phosphorylation of Akt and neuronal survival after transient focal cerebral ischemia in mice. J Cereb Blood Flow Metab 21(12):1442-1450

45. Park KK, Liu K, Hu Y, Smith PD, Wang C, Cai B, Xu B, Connolly L, Kramvis I, Sahin M, He Z (2008) Promoting axon regeneration in the adult CNS by modulation of the PTEN/mTOR pathway. Science 322(5903):963-966

46. Sun F, Park KK, Belin S, Wang D, Lu T, Chen G, Zhang K, Yeung C, Feng G, Yankner BA, He Z (2011) Sustained axon regeneration induced by co-deletion of PTEN and SOCS3. Nature 480(7377):372-375

47. Liu L, Sun T, Xin F, Cui W, Guo J, Hu J (2017) Nerve growth factor protects against alcohol-induced neurotoxicity in PC12 cells viaPI3K/Akt/mTOR pathway. Alcohol Alcohol 52(1):12-18

48. Nijboer CH, Bonestroo HJ, Zijlstra J, Kavelaars A, Heijnen CJ (2013) Mitochondrial JNK phosphorylation as a novel therapeutic target to inhibit neuroinflammation and apoptosis after neonatal ischemic brain damage. Neurobiol Dis 54:432-444 
49. Aubert N, Falluel-Morel A, Vaudry D, Xifro X, Rodriguez-Alvarez J, Fisch C, de Jouffrey S, Lebigot JF, Fournier A, Vaudry H, Gonzalez BJ (2006) PACAP and C2-ceramide generate different AP-1 complexes through a MAP-kinase-dependent pathway: involvement of c-Fos in PACAP-induced Bcl-2 expression. J Neurochem 99(4):1237-1250

50. Dohi K, Mizushima H, Nakajo S et al (2003) Pituitary adenylate cyclase-activating polypeptide (PACAP) prevents hippocampal neurons from apoptosis by inhibiting JNK/SAPK and p38 signal transduction pathways. Regul Pept 109(1-3):83-88

51. Falluel-Morel A, Aubert N, Vaudry D, Basille M, Fontaine M, Fournier A, Vaudry H, Gonzalez BJ (2004) Opposite regulation of the mitochondrial apoptotic pathway by $\mathrm{C} 2$-ceramide and PACAP through a MAP-kinase-dependent mechanism in cerebellar granule cells. J Neurochem 91(5):1231-1243

52. Meng X, Sun G, Ye J, Xu H, Wang H, Sun X (2014) Notoginsenoside R1-mediated neuroprotection involves estrogen receptordependent crosstalk between Akt and ERK1/2 pathways: a novel mechanism of Nrf2/ARE signaling activation. Free Radic Res 48(4):445-460

53. Sun DS, Chang HH (2003) Differential regulation of JNK in caspase-3-mediated apoptosis of MPP(+)-treated primary cortical neurons. Cell Biol 27(9):769-777

54. Chen W, Ma Q, Suzuki H, Hartman R, Tang J, Zhang JH (2011) Osteopontin reduced hypoxiaischemia neonatal brain injury by suppression of apoptosis in a ratpup model. Stroke 42(3):764-769

55. Monaco CM, Gebhardt KM, Chlebowski SM, Shaw KE, Cheng JP, Henchir JJ, Zupa MF, Kline AE (2014) Combined therapeutic regimen of buspirone and environmental enrichment is more efficacious than either alone in enhancing spatial learning in brain-injured pediatric rats. J Neurotrauma 31(23):1934-1941

56. Ohlsson AL, Johansson BB (1995) Environment influences functional outcome of cerebral infarction in rats. Stroke 26(4):6449

57. Feeney DM, Gonzalez A, Law WA (1982) Amphetamine, haloperidol and experience interact to affect the rate of recovery after motor cortex injuries. Science 217(4562):855-857

58. Fathali N, Ostrowski RP, Lekic T, Jadhav V, Tong W, Tang J, Zhang JH (2010) Cyclooxygenase-2 inhibition provides lasting protection against neonatal hypoxic-ischemic brain injury. Crit Care Med 38(2):572-578

59. Chen D, Tang J, Khatibi NH, Zhu M, Li Y, Wang C, Jiang R, Tu L, Wang S (2011) Treatment with Z-ligustilide, a component of Angelica sinensis, reduces brain injury after a subarachnoid hemorrhage in rats. J Pharmacol Exp Ther 337(3):663-672

60. Cheskis BJ, Greger J, Cooch N, McNally C, Mclarney S, Lam HS, Rutledge S, Mekonnen B, Hauze D, Nagpal S, Freedman LP (2008) MNAR plays an important role in ERa activation of Src/MAPK and PI3K/Akt signaling pathways. Steroids 73(9-10):901-905

61. Fitzpatrick JL, Mize AL, Wade CB, Harris JA, Shapiro RA, Dorsa DM (2002) Estrogen-mediated neuroprotection against beta-amyloid toxicity requires expression of estrogen receptor alpha or beta and activation of the MAPK pathway. J Neurochem 82(3):674-682

62. Greger JG, Fursov N, Cooch N, McLarney S, Freedman LP, Edwards DP, Cheskis BJ (2007) Phosphorylation of MNAR promotes estrogen activation of phosphatidylinositol 3-kinase. Mol Cell Biol 27(5):1904-1913

63. Sun M, Paciga JE, Feldman RI, Yuan Z, Coppola D, Lu YY, Shelley SA, Nicosia SV, Cheng JQ (2001) Phosphatidylinositol-3-OH Kinase (PI3K)/AKT2, activated in breast cancer, regulates and is induced by estrogen receptor alpha (ERalpha) via interaction between ERalpha and PI3K. Cancer Res 61(16):5985-5991

64. Yang JL, Chen WY, Chen YP, Kuo CY, Chen SD (2016) Activation of GLP-1 receptor enhances neuronal base excision repair via PI3KAKT-induced expression of apurinic/apyrimidinic endonuclease 1. Theranostics 6(12):2015-2027
65. Engler-Chiurazzi EB, Brown CM, Povroznik JM, Simpkins JW (2017) Estrogens as neuroprotectants: estrogenic actions in estrogenic actions in the context of cognitive aging and braininjury. Prog Neurobiol 157:188-211

66. Weiland NG, Orikasa C, Hayashi S, McEwen BS (1997) Distribution and hormone regulation of estrogen receptor immunoreactive cells in the hippocampus of male and female rats. J Comp Neuro 1388:603-612

67. Kritzer MF (2002) Regional, laminar, and cellular distribution of immunoreactivity for ER alpha and ER beta in the cerebral cortex of hormonally intact, adult male and female rats. Cereb Cortex $12: 116-128$

68. Shin JA, Choi JH, Choi YH, Park EM (2011) Conserved aquaporin 4 levels associated with reduction of brain edema are mediated by estrogen in the ischemic brain after experimental stroke. Biochim Biophys Acta 1812(9):1154-1163

69. Su P, Du S, Li H, Li Z, Xin W et al (2016) Notoginsenoside R1 inhibits oxidized low-density lipoprotein induced inflammatory cytokines production in human endothelial EA.hy926 cells. Eur J Pharmacol 770:9-15

70. Huang G, Zou B, Lv J, Li T, Huai G, Xiang S, Lu S, Luo H, Zhang Y, Jin Y, Wang Y (2017) Notoginsenoside R1 attenuates glucoseinduced podocyte injury via the inhibition of apoptosis and the activation of autophagy through the PI3K/Akt/mTOR signaling pathway. Int J Mol Med 39:559-568

71. Li Z, Li H, Zhao C, Lv C, Zhong C, Xin W, Zhang W (2015) Protective effect of notoginsenoside R1 on an APP/PS1 mouse model of Alzheimer's disease by up-regulating insulin degrading enzyme and inhibiting $A \beta$ accumulation. CNS Neurol Disord Drug Targets 14(3):360-369

72. Hossain MA (2008) Hypoxic-ischemic injury in neonatal brain: involvement of a novel neuronal molecule in neuronal cell death and potential target for neuroprotection. Int J Dev Neurosci 26(1):93-101

73. Nakajima T, Iwabuchi S, Miyazaki H et al (2004) Preconditioning prevents ischemia-induced neuronal death through persistent Akt activation in the penumbra region of the rat brain. J Vet Med Sci 66:521-527

74. Pastor MD, Garcia-Yebenes I, Fradejas N, Perez-Ortiz JM, MoraLee S, Tranque P, Moro MA, Pende M, Calvo S (2009) mTOR/S6 kinase pathway contributes to astrocyte survival during ischemia. J Biol Chem 284:22067-22078

75. Chen HJ, Xiong T, Qu Y, Zhao FY, Ferriero D, Mu DZ (2012) mTOR activates hypoxia-inducible factor- $1 \alpha$ and inhibits neuronal apoptosis in the developing rat brain during the early phase after hypoxia-ischemia. Neurosci Lett 507:118-123

76. Park KK, Liu K, Hu Y, Smith PD, Wang C, Cai B, Xu B, Connolly L, Kramvis I, Sahin M et al (2008) Promoting axon regeneration in the adult CNS by modulation of the PTEN/mTOR pathway. Science 322:963-966

77. Ezquer ME, Valdez SR, Seltzer AM, Jahn GA (2008) Advancement of reproductive senescence and changes in the early expression of estrogen, progesterone and $\mu$-opioid receptors induced by neonatal hypoxia in the female rat. Brain Res 1214:73-83

78. Kraczkowski JJ, Semczuk M (2000) Sex and changes in mu-opioid receptor density under hypoxia. Ginekol Pol 71:927-930

79. Simoncini T, Hafezi-Moghadam A, Brazil DP, Ley K, Chin WW, Liao JK (2000) Interaction of oestrogen receptor with the regulatory subunit of phosphatidylinositol-3-OH kinase. Nature 407(6803):538-541

80. Santen RJ, Song RX, Zhang Z, Kumar R, Jeng MH, Masamura A, Lawrence J Jr, Berstein L, Yue W (2005) Long-term estradiol deprivation in breast cancer cells up-regulates growth factor signaling and enhances estrogen sensitivity. Endocr Relat Cancer 12:S61-S73

81. Dhillon SS, Belsham DD (2011) Estrogen inhibits NPY secretion through membrane-associated estrogen receptor (ER)- $\alpha$ in clonal, immortalized hypothalamic neurons. Int J Obes 35(2):198-207 
82. Meitzen J, Mermelstein PG (2011) Estrogen receptors stimulate brain region specific metabotropic glutamate receptors to rapidly initiate signal transduction pathways. J Chem Neuroanat 42(4):236-241
83. Bains M, Roberts JL (2016) Estrogen protects against dopamine neuron toxicity in primary mesencephalic cultures through an indirect P13K/Akt mediated astrocyte pathway. Neurosci Lett 610:79-85 